\title{
The Impact of Water and Ethanolic Extracts of Propolis on Fatty Acid Changes of Sardine Fillets
}

\begin{tabular}{|c|c|}
\hline \multicolumn{2}{|c|}{$\begin{array}{l}\text { Aykut Burgut }{ }^{1, a, *} \\
{ }^{I} \text { Department of Animal Science Technology, Faculty of Agriculture, University of Cukurova, } 01330 \text { Adana, Turkey. } \\
{ }^{*} \text { Corresponding author }\end{array}$} \\
\hline A R T I C L E I N F O & A B S T R A C T \\
\hline $\begin{array}{l}\text { Keywords: } \\
\text { Propolis extract } \\
\text { Sardine } \\
\text { Eicosapentaenoic acid } \\
\text { Docosahexaenoic acid } \\
\text { Polyunsaturated fatty acids }\end{array}$ & $\begin{array}{l}\text { The polyunsaturated fatty acids (PUFA) of lipids in muscle of sardine were known as unstable to } \\
\text { oxidation. Propolis is a natural compounds produced by honey bees from substances collected from } \\
\text { parts of plants, buds, and exudates. Nowadays, the particular attention has been attributed to } \\
\text { propolis underlying their antioxidant properties due to the presence of the flavonoids, phenolic } \\
\text { acids, and ethers. The impact of water and ethanolic extract of propolis at doses of } 0.4 \text { and } 0.8 \% \text { on } \\
\text { fatty acid changes of vacuum packaged sardine fillets were investigated during chill storage. The } \\
\text { main fatty acids in sardine fillets were palmitic acid (C16:0), palmitoleic acid (C16:1), myristic acid } \\
(\mathrm{C} 14: 0) \text {, eicosapentaenoic acid (EPA, C20:5n3) and docosahexaenoic acid (DHA, C22:6 n3), } \\
\text { respectively. There was no significant differences in EPA and DHA between control and treated } \\
\text { groups during storage apart from } 4 \text { and } 11^{\text {th }} \text { day. Initial n6/n } 3 \text { ratio in sardine fillet was } 0.17 \text {. Group } \\
\text { treated with } 0.4 \text { propolis ethanolic extract comprised lower n } 6 / \mathrm{n} 3 \text { ratio at } 4,11 \text { and } 13^{\text {th }} \text { days than } \\
\text { that of other groups. Although the effects of propolis extracts on fatty acid composition of fish were } \\
\text { variable, application of propolis ethanolic and water extracts on fish fillets at doses of } 0.8 \% \text { had a } \\
\text { positive impact due to lead a better oxidative stability of PUFA content. }\end{array}$ \\
\hline
\end{tabular}

\section{Introduction}

Certain fatty acids, specifically polyunsaturated fatty acids (PUFA), can only be biosynthesised by certain species of phytoplankton and macroalgae and can be traced as essential dietary components to higher trophic levels such as zooplankton (Van der Meeren et al., 2008) and fish (Metillo and Aspiras-Eya, 2014). Thus, fish meat is considered as abundant source of long chain PUFA and valuable nutritional components (Ali et al., 2019). The fatty acids composition of fish lipids, especially of PUFA, is species-specific and is correlated to various factors, including dietary, geographic and environmental factors, reproductive season, fishery period, etc. (Passi et al., 2002).

Lipid oxidation is a chemical change that can decrease the quality of the meat products because it generates undesirables odor, color, smell and flavor reducing the nutritional value (Gutiérrez -Cortés and Suarez-Mahecha, 2014). Lipid oxidation are main reasons of quality deterioration in the meat products throughout storage which has near about estimate price of the industry loss over $\$ 700$ million annually (Ali et al., 2019). The PUFA in the lipids of sardines were stable to cooking, but unstable to oxidation during storage in a refrigerator. The PUFA of lipids in the dark meat of sardine were extremely unstable to oxidation (Yamamoto and Imose, 1989). Marked decreases in unsaturated fatty acids, especially eicosapentaenoic acid (EPA; C20:5 n-3) and docosahexaenoic acid (DHA; C22:6n-3) were observed as the storage time increased (Chaijan et al., 2006). In order to inhibit lipid oxidation of foods, some preservatives additives used as antioxidants and have proved be unhealthy to the consumers. Therefore, it is important to develop natural products with antioxidant activity. Various studies have reported that ethanolic propolis extracts applied in different doses (0.01-2\%) positively affected the oxidation stability of meat and meat products (Kročko et al., 2014; Vargas-Sánchez et al., 2014).

Propolis is a bee product resulting from the collection of resin from different parts of plants and has a strong antioxidant activity (Touzani et al., 2018). More than 240 propolis-containing substances have been identified (Huang et al., 2014), many of them with anti-carcinogenic, anti-inflammatory, antimicrobial and antioxidant 
properties (Hernandez Zarate et al., 2018). It contains a large spectrum of compounds: polyphenols, flavonoids, and other organic and inorganic molecules (Boufadi et al., 2014). Among the broad biological properties of propolis, antioxidant capacity is one of the most important properties, which contributes greatly to prevent certain illnesses, including cardiovascular diseases, diabetes and cancer (Zheng et al., 2017). Strong antioxidant activity of propolis is due to their large amounts of antioxidative compounds, such as caffeic acid, ferulic acid and caffeic acid phenethyl ester (Ahn et al., 2007). In this study, the effect of water and propolis extracts on fatty acids changes of vacuum packaged sardine was investigated during refrigerated storage for 15 th day.

\section{Material and Method}

\section{Propolis Extracts}

Propolis from Apis mellifera was collected from Adana, Turkey, in April 2018. In order to improve extraction efficiency, propolis was pulverized prior to extraction. For ethanolic and aqueous extraction, $70 \%$ ethanol and $100 \%$ water were added to the powder propolis, respectively. They were then placed in daily shakable containers in the dark. They were stored in a dark place at $4^{\circ} \mathrm{C}$ until application.

\section{Preparation of Fish Samples}

The sardine (Sardine aurita) was caught using a gill net (April 2018) in Mersin Bay, Turkey. Fish were kept in boxes with ice on board after catching and delivered to the laboratory in $14 \mathrm{~h}$. The fish were immediately gutted and filleted without skin removal. After that, they were washed with sterile water and the fillets divided into 5 groups. The first group was an untreated with propolis extract as control. The treatment groups contained propolis aqueous (PW) or ethanolic extract (PE) at a dose of 0.4 or $0.8 \%$ of fish weight for 4 minute. Each group was vacuum packaged in pouches with a polyamide base (Polinas, Manisa, Turkey) at -0.1 bar with $20 \mathrm{~s}$ sealing (Reepack RV50, Seriate BG, Italy) and stored at $3 \pm 1^{\circ} \mathrm{C}$ for $15^{\text {th }}$ day. Fish fillets were analysed at $0,4,7,9,11,13$ and $15^{\text {th }}$ days for each treatment.

\section{Fatty Acid Analyses}

Lipid content was determined by the method of Bligh and Dyer (1959). Methyl esterification of lipid samples were made according to method of Ichihara et al. (1996), by using $2 \mathrm{M} \mathrm{KOH}$ in methanol and n-heptane with minor modifications. Briefly, twenty $\mathrm{mg}$ of extracted oil was dissolved in $2 \mathrm{ml} \mathrm{n}$-heptane followed by $4 \mathrm{ml}$ of $2 \mathrm{M}$ methanolic $\mathrm{KOH}$. After centrifugation at 4,000 rpm for 10 min, the n-heptane layer was taken for gas chromatography analyses.

\section{Gas Chromatographic (GC) Conditions}

The fatty acid composition was determined by a GC Clarus 500 (Perkin Elmer, Shelton, CT, USA) equipped with a flame ionization detector and a fused silica capillary SGE column (30 m0.32 mm ID0.25 mm BP20 0.25 UM; (Perkin Elmer, Shelton, CT, USA). The oven temperature was $140^{\circ} \mathrm{C}$ held for $5 \mathrm{~min}$, raised to $200^{\circ} \mathrm{C}$ at a rate of $4^{\circ} \mathrm{C} / \mathrm{min}$ and then to $220^{\circ} \mathrm{C}$ at a rate of $1^{\circ} \mathrm{C} / \mathrm{min}$, while the injector and detector temperatures were set at $220^{\circ} \mathrm{C}$ and $280^{\circ} \mathrm{C}$, respectively. The sample size was $1 \mathrm{ml}$ and the carrier gas was controlled at 16 ps. The split used was 1:50. Fatty acids were analysed by comparing the retention times of FAME (catalogue number 18919; Supelco) with the standard 37- component fatty acid methyl esters (FAME) mixture. Two replicate gas chromatographic analyses were performed and the results expressed in the gas chromatographic area percentage.

\section{Statistical Analysis}

IBM SPSS Statistic 20 was used to determine for statistical analysis. Data were subjected to analysis Duncan's multiple range tests with a significant difference at $\mathrm{P}<0.05$.

\section{Result and Discussion}

Changes in fatty acids of sardine treated with or without propolis extracts during storage were given Tables 1-4. Main saturated fatty acids (SFA) found in sardine were palmitic acid (C16:0), myristic acid (C14:0) and stearic acid (C18:0), comprising initial value of $21.52 \%, 12.54$, and $5.3 \%$. The principal constituent of SFA was also reported by De Leonardis and Macciola (2004) as palmitic acid, followed by myristic and stearic acids. Palmitic acid content of fish fillets did not change significantly between control and treated group during storage apart from at 4,11 and $13^{\text {th }}$ days. In the presence of $0.4 \%$ ethanolic propolis extract, myristic acid content in fish fillet significantly decreased at 4,13 and $15^{\text {th }}$ days, whereas higher content of myristic acid was observed in the presence of $0.8 \%$ propolis water extract at $4^{\text {th }}$ day compared to control group. Total SFA content of sardine was 42.49 and $41.83 \%$ at 0 and $15^{\text {th }}$ days, respectively (Table 1). Total SFA content of fish did not vary significantly for control during chill storage. Total SFA content of sardine decreased for $0.4 \mathrm{PE}$ group at 4 and $15^{\text {th }}$ day and for $0.8 \mathrm{PW}$ at $9^{\text {th }}$ day, whereas increased at $9^{\text {th }}$ day for $0.8 \mathrm{PE}$ and $14^{\text {th }}$ day for $0.4 \mathrm{PW}$ group $(\mathrm{P}<0.05)$. There was not significant differences in total SFA content among groups at 4, 7 and $15^{\text {th }}$ days. Total SFA level considerably higher by addition of 0.8 and $0.4 \%$ propolis extract at 9 and $13^{\text {th }}$ day, respectively. Decreases in total SFA level was also noticed at $11^{\text {th }}$ day for $0.8 \mathrm{PE}$ group.

Palmitoleic (C16:1, 14.05\%), oleic (C18:1n9, 4.88\%) and cis-vaccenic acid (C18:1n7, 4.07\%) were main MUFA found in fresh sardine fillet (Table 2). Application of extract on fish fillets did not affect palmitoleic acid content in fish flesh apart from $0.4 \mathrm{PE}$ group at 4,13 and $15^{\text {th }}$ days and $0.8 \mathrm{PW}$ group at $13^{\text {th }}$ days. Oleic acid level was the highest by ethanolic propolis extract at 4 and $15^{\text {th }}$ days. There was not significant changes in vaccenic acid level among group $(\mathrm{P}>0.05)$. Total MUFA content of sardine fillet was $24.37 \%$ at the beginning of storage and did not change significantly during storage for control and groups treated with ethanolic extract of propolis. There were also not significant differences in MUFA content among group at 4 and $15^{\text {th }}$ day $(\mathrm{P}>0.05)$. MUFA was the highest concentration at $7^{\text {th }}$ days and the lowest at $9^{\text {th }}$ days for $0.4 \mathrm{~W}$ group compared to other groups. At 11 day and $13^{\text {th }}$ day, $0.8 \mathrm{PW}$ and $0.4 \mathrm{PE}$ group had considerably lower total MUFA (22.48 vs 23.17) content than control group (24.56 vs. $25.11 \%$ ), respectively. 
Table 1. Changes in SFA (\%) content of sardine treated with/without water or ethanolic extract of propolis during chill storage.

\begin{tabular}{|c|c|c|c|c|c|c|c|c|c|c|c|c|c|}
\hline Days & Groups & C8:0 & C12:0 & C13:0 & C14:0 & C15:0 & C16:0 & C17:0 & C18:0 & C20:0 & C22:0 & C24:0 & SFA \\
\hline \multirow{2}{*}{0} & \multirow{2}{*}{ C } & $0.18^{x}$ & 0.05 & 0.02 & 12.54 & 0.63 & 21.52 & 0.98 & 5.3 & 0.62 & 0.53 & 0.13 & $42.49^{\mathrm{A}}$ \\
\hline & & $0.01^{\mathrm{y}}$ & 0.00 & 0.00 & 0.45 & 0.01 & 0.33 & 0.07 & 0.42 & 0.01 & 0.01 & 0.00 & 0.23 \\
\hline \multirow{10}{*}{4} & \multirow{2}{*}{ C } & $0.04 b$ & \multirow{2}{*}{ ND } & \multirow{2}{*}{ ND } & $11.76^{\mathrm{b}}$ & $0.42^{\mathrm{a}}$ & $22.36^{\mathrm{a}}$ & $0.88^{\mathrm{a}}$ & $6.10^{\mathrm{a}}$ & $0.39^{\mathrm{a}}$ & $0.57^{\mathrm{a}}$ & $0.15^{\mathrm{a}}$ & $42.68^{\mathrm{aA}}$ \\
\hline & & 0.00 & & & 0.13 & 0.31 & 2.21 & 0.16 & 0.08 & 0.01 & 0.01 & 0.00 & 2.57 \\
\hline & \multirow{2}{*}{$0.4 \mathrm{PE}$} & $0.18^{\mathrm{a}}$ & $0.05^{\mathrm{a}}$ & \multirow{2}{*}{ ND } & $11.16^{\mathrm{c}}$ & $0.57^{\mathrm{a}}$ & $20.32^{\mathrm{ab}}$ & $0.87^{\mathrm{a}}$ & $5.51^{\mathrm{b}}$ & $0.32^{\mathrm{b}}$ & $0.56^{\mathrm{a}}$ & $0.08^{\mathrm{d}}$ & $39.64^{\mathrm{aC}}$ \\
\hline & & 0.01 & 0.03 & & 0.45 & 0.01 & 0.12 & 0.04 & 0.19 & 0.00 & 0.01 & 0.00 & 0.21 \\
\hline & \multirow{2}{*}{$0.4 \mathrm{PW}$} & \multirow{2}{*}{ ND } & \multirow{2}{*}{ ND } & & $12.57^{\mathrm{ab}}$ & $0.63^{\mathrm{a}}$ & $19.52^{\mathrm{b}}$ & $0.99^{\mathrm{a}}$ & $4.78^{c}$ & $0.28^{c}$ & $0.57^{\mathrm{a}}$ & $0.12^{\mathrm{c}}$ & $39.42^{\mathrm{aB}}$ \\
\hline & & & & ND & 0.09 & 0.01 & 0.22 & 0.01 & 0.06 & 0.01 & 0.01 & 0.01 & 0.21 \\
\hline & & $0.19^{\mathrm{a}}$ & $0.08^{\mathrm{a}}$ & $0.03^{\mathrm{a}}$ & $12.28^{\mathrm{b}}$ & $0.59^{\mathrm{a}}$ & $19.69^{\mathrm{ab}}$ & $0.96^{\mathrm{a}}$ & $4.97^{c}$ & $0.32^{\mathrm{b}}$ & $0.58^{\mathrm{a}}$ & $0.14^{\mathrm{b}}$ & $39.81^{\mathrm{aB}}$ \\
\hline & $0.8 \mathrm{PE}$ & 0.06 & 0.00 & 0.00 & 0.17 & 0.01 & 0.3 & 0.03 & 0.29 & 0.02 & 0.01 & 0.00 & 0.73 \\
\hline & & $0.09^{\mathrm{b}}$ & $0.07^{\mathrm{a}}$ & $0.03^{\mathrm{a}}$ & $11.87^{\mathrm{a}}$ & $0.64^{\mathrm{a}}$ & $21.33^{\mathrm{ab}}$ & $0.9^{\mathrm{a}}$ & $5.72^{\mathrm{ab}}$ & $0.37^{\mathrm{a}}$ & $0.56^{\mathrm{a}}$ & $0.14^{\mathrm{b}}$ & $41.70^{\mathrm{aAB}}$ \\
\hline & $8 \mathrm{PV}$ & 0.00 & 0.00 & 0.00 & 0.02 & 0.01 & 0.44 & 0.00 & 0.31 & 0.03 & 0.01 & 0.00 & 0.81 \\
\hline & C & $0.02^{\mathrm{a}}$ & $0.04^{\mathrm{ab}}$ & & $12.57^{\mathrm{a}}$ & $0.67^{\mathrm{a}}$ & $21.04^{\mathrm{a}}$ & $0.98^{\mathrm{a}}$ & $5.25^{\mathrm{a}}$ & $0.34^{\mathrm{a}}$ & $0.53^{\mathrm{b}}$ & $0.08^{\mathrm{b}}$ & $41.69^{\mathrm{aA}}$ \\
\hline & C & 0.01 & 0.00 & ND & 0.11 & 0.04 & 0.97 & 0.01 & 0.01 & 0.03 & 0.0 & 0.01 & 1.22 \\
\hline & IPE & $0.21^{\mathrm{a}}$ & $0.06^{\mathrm{a}}$ & $0.03^{\mathrm{b}}$ & $12.22^{\mathrm{a}}$ & $0.62^{\mathrm{a}}$ & $20.14^{\mathrm{a}}$ & $0.91^{\mathrm{a}}$ & $5.20^{\mathrm{a}}$ & $0.32^{\mathrm{ab}}$ & $0.56^{\mathrm{a}}$ & $0.13^{\mathrm{ab}}$ & $40.38^{\mathrm{aAB}}$ \\
\hline & $\mathrm{PE}$ & 0.04 & 0.00 & 0.00 & 0.37 & 0.01 & 0.32 & 0.06 & 0.38 & 0.01 & 0.00 & 0.01 & 0.30 \\
\hline & & $0.14^{\mathrm{b}}$ & $0.07^{\mathrm{a}}$ & $0.03^{\mathrm{b}}$ & $12.3^{\mathrm{a}}$ & $0.60^{\mathrm{a}}$ & $20.10^{\mathrm{a}}$ & $1.01^{\mathrm{a}}$ & $5.07^{\mathrm{a}}$ & $0.28^{\mathrm{b}}$ & $0.57^{\mathrm{a}}$ & $0.11^{\mathrm{ab}}$ & $40.27^{\mathrm{aAB}}$ \\
\hline 1 & & 0.00 & 0.00 & 0.00 & 0.6 & 0.01 & 0.14 & 0.07 & 0.11 & 0.01 & 0.01 & 0.00 & 0.71 \\
\hline & 10 & ND & $N$ & $\mathrm{~N}$ & $12.32^{\mathrm{a}}$ & $0.65^{\mathrm{a}}$ & $21.42^{\mathrm{a}}$ & $1.03^{\mathrm{a}}$ & $5.18^{\mathrm{a}}$ & $0.33^{\mathrm{ab}}$ & $0.55^{\mathrm{ab}}$ & $0.13^{\mathrm{ab}}$ & $41.59^{\mathrm{aB}}$ \\
\hline & & & & & 0.88 & 0.01 & 1.65 & 0.01 & 0.24 & 0.04 & 0.01 & 0.02 & 1.06 \\
\hline & 10 & $0.22^{\mathrm{a}}$ & 0.03 & $0.07^{\mathrm{a}}$ & $11.71^{\mathrm{a}}$ & $0.66^{\mathrm{a}}$ & $21.27^{\mathrm{a}}$ & $0.92^{\mathrm{a}}$ & $5.43^{\mathrm{a}}$ & $0.33^{\mathrm{ab}}$ & $0.53^{b}$ & $0.15^{\mathrm{a}}$ & $41.31^{\mathrm{aAB}}$ \\
\hline & & 0.01 & 0.00 & 0.00 & 0.17 & 0.04 & 0.90 & 0.04 & 0.23 & 0.01 & 0.00 & 0.03 & 1.36 \\
\hline & & $0.17^{b}$ & $0.07^{\mathrm{a}}$ & $0.03^{\mathrm{a}}$ & $12.04^{\mathrm{a}}$ & $0.61^{\mathrm{a}}$ & $21.00^{\mathrm{ab}}$ & $0.90^{\mathrm{ab}}$ & $5.71^{\mathrm{b}}$ & $0.34^{\mathrm{a}}$ & $0.54^{\mathrm{a}}$ & $0.14^{\mathrm{ab}}$ & $41.34^{\mathrm{bA}}$ \\
\hline & C & 0.01 & 0.00 & 0.00 & 0.41 & 0.04 & 0.19 & 0.01 & 0.15 & 0.02 & 0.01 & 0.01 & 0.18 \\
\hline & & $0.13^{c}$ & $0.08^{\mathrm{a}}$ & $0.00^{\mathrm{b}}$ & $11.91^{\mathrm{a}}$ & $0.61^{\mathrm{a}}$ & $20.27^{\mathrm{b}}$ & $0.96^{\mathrm{a}}$ & $5.21^{\mathrm{b}}$ & $0.45^{\mathrm{a}}$ & $0.57^{\mathrm{a}}$ & $0.13^{\mathrm{b}}$ & $40.30^{\mathrm{bAB}}$ \\
\hline & $0.4 \mathrm{PE}$ & 0.01 & 0.01 & 0.00 & 0.76 & 0.04 & 0.41 & 0.04 & 0.47 & 0.25 & 0.00 & 0.01 & 0.3 \\
\hline & & $0.25^{\mathrm{a}}$ & $0.07^{\mathrm{a}}$ & $0.05^{\mathrm{a}}$ & $11.24^{\mathrm{a}}$ & $0.59^{\mathrm{a}}$ & $20.78^{\mathrm{ab}}$ & $0.83^{b}$ & $5.71^{\mathrm{b}}$ & $0.34^{\mathrm{a}}$ & $0.54^{\mathrm{a}}$ & $0.13^{\mathrm{b}}$ & $40.46^{\mathrm{bAB}}$ \\
\hline 9 & $0.4 \mathrm{H}$ & 0.02 & 0.00 & 0.00 & 0.06 & 0.02 & 0.49 & 0.04 & 0.24 & 0.02 & 0.02 & 0.00 & 0.25 \\
\hline & $0 \mathrm{SI}$ & $0.23^{\mathrm{a}}$ & $0.05^{\mathrm{b}}$ & $0.03^{\mathrm{a}}$ & $11.35^{\mathrm{a}}$ & $0.63^{\mathrm{a}}$ & $24.22^{\mathrm{a}}$ & $0.82^{b}$ & $7.12^{\mathrm{a}}$ & $0.38^{\mathrm{a}}$ & $0.56^{\mathrm{a}}$ & $0.16^{\mathrm{a}}$ & $45.53^{\mathrm{aA}}$ \\
\hline & $0.8 \mathrm{PE}$ & 0.00 & 0.00 & 0.00 & 0.3 & 0.04 & 2.86 & 0.02 & 0.13 & 0.03 & 0.06 & 0.01 & 3.00 \\
\hline & | & $0.19^{b c}$ & $0.07^{\mathrm{a}}$ & $0.00^{\mathrm{b}}$ & $11.88^{\mathrm{a}}$ & $0.58^{\mathrm{a}}$ & $20.38^{\mathrm{b}}$ & $0.95^{\mathrm{a}}$ & $5.30^{\mathrm{b}}$ & $0.59^{\mathrm{a}}$ & $0.55^{\mathrm{a}}$ & $0.14^{\mathrm{ab}}$ & $40.60^{\mathrm{bB}}$ \\
\hline & $0.8 \mathrm{PW}$ & 0.01 & 0.00 & 0.00 & 0.62 & 0.02 & 0.28 & 0.05 & 0.45 & 0.33 & 0.03 & 0.00 & 0.88 \\
\hline & $\Gamma_{2}$ & $0.16^{\mathrm{c}}$ & $0.07^{\mathrm{a}}$ & $\mathrm{NI}$ & 11.1 & $0.62^{\mathrm{a}}$ & $22.32^{\mathrm{ab}}$ & $0.95^{\mathrm{a}}$ & $5.75^{b}$ & $0.28^{c}$ & $0.58^{\mathrm{a}}$ & $0.16^{\mathrm{a}}$ & $42.02^{\mathrm{abA}}$ \\
\hline & C & 0.00 & 0.00 & $\mathrm{NI}$ & 0.93 & 0.0 & 1.22 & 0. & 0.11 & 0.00 & 0.00 & 0.00 & 0.30 \\
\hline & & $0.19^{\mathrm{bc}}$ & $0.08^{\mathrm{a}}$ & & $12.18^{\mathrm{a}}$ & $0.58^{\mathrm{b}}$ & $20.67^{b c}$ & $0.94^{\mathrm{a}}$ & $5.34^{\mathrm{bc}}$ & $0.32^{\mathrm{b}}$ & $0.56^{\mathrm{ab}}$ & $0.14^{\mathrm{abc}}$ & $41.01^{\mathrm{bcAB}}$ \\
\hline & $+\mathrm{H}$ & 0.01 & 0.00 & & & 0.01 & 0.00 & 0.01 & 0.01 & 0.01 & 0. & 0.01 & 0.56 \\
\hline 11 & & $0.23^{\mathrm{b}}$ & $0.07^{\mathrm{a}}$ & & $12.22^{\mathrm{a}}$ & $0.63^{\mathrm{a}}$ & $20.79^{\mathrm{abc}}$ & $0.94^{\mathrm{a}}$ & $5.45^{\mathrm{bc}}$ & $0.35^{\mathrm{b}}$ & $0.54^{\mathrm{ab}}$ & $0.13^{b c}$ & $41.36^{\mathrm{bcA}}$ \\
\hline 11 & & 0.01 & 0.00 & $N$ & 0.48 & 0.01 & 0.68 & 0.06 & 0.44 & 0.02 & 0.00 & 0.01 & 0.63 \\
\hline & & $0.20^{\mathrm{b}}$ & $0.07^{\mathrm{a}}$ & NI & $12.00^{\mathrm{a}}$ & $0.62^{\mathrm{a}}$ & $20.17^{\mathrm{c}}$ & $0.97^{\mathrm{a}}$ & $5.09^{c}$ & $0.33^{\mathrm{b}}$ & $0.57^{\mathrm{ab}}$ & $0.12^{\mathrm{c}}$ & $40.15^{\mathrm{cB}}$ \\
\hline & & 0.02 & 0.00 & & 0.37 & 0.01 & 0.23 & 0.03 & 0.04 & 0.02 & 0.01 & 0.00 & 0.13 \\
\hline & & $0.31^{\mathrm{a}}$ & $0.05^{\mathrm{a}}$ & $\mathrm{NI}$ & $10.38^{\mathrm{b}}$ & $0.64^{\mathrm{a}}$ & $22.62^{\mathrm{a}}$ & $0.77^{b}$ & $6.84^{\mathrm{a}}$ & $0.59^{\mathrm{a}}$ & $0.52^{\mathrm{c}}$ & $0.15^{\mathrm{ab}}$ & $42.89^{\mathrm{aA}}$ \\
\hline & & 0.00 & 0.00 & NDD & 0.00 & 0.00 & 0.71 & 0.00 & 0.14 & 0.00 & 0.04 & 0.00 & 0.81 \\
\hline & IC & $0.19^{\mathrm{b}}$ & 0.07 & $0.03^{\mathrm{a}}$ & $12.86^{\mathrm{a}}$ & $0.63^{\mathrm{a}}$ & $20.68^{\mathrm{bc}}$ & $0.98^{\mathrm{a}}$ & $5.06^{\mathrm{c}}$ & $0.32^{\mathrm{a}}$ & $0.56^{\mathrm{a}}$ & $0.16^{\mathrm{a}}$ & $41.51^{\mathrm{bcA}}$ \\
\hline & |c & 0.01 & 0.00 & 0.00 & 0.87 & 0.02 & 0.47 & 0.09 & 0.23 & 0.01 & 0.01 & 0.01 & 0.20 \\
\hline & & $0.17^{b}$ & 0.06 & & $11.14^{\mathrm{b}}$ & $0.58^{b}$ & $25.35^{\mathrm{a}}$ & $0.96^{\mathrm{a}}$ & $8.10^{\mathrm{a}}$ & $0.28^{\mathrm{a}}$ & $0.58^{\mathrm{a}}$ & $0.03^{\mathrm{e}}$ & $47.23^{\mathrm{aA}}$ \\
\hline & 0. & 0.00 & 0.00 & & 0.18 & 0.02 & & 0.06 & 0.26 & 0.01 & 0.01 & 0.00 & 1.74 \\
\hline 13 & & $0.19^{b}$ & 0.07 & $0.03^{\mathrm{a}}$ & $12.53^{\mathrm{ab}}$ & $0.61^{\mathrm{ab}}$ & $19.71^{\mathrm{c}}$ & $0.74^{\mathrm{a}}$ & $4.93^{c}$ & $0.28^{\mathrm{a}}$ & $0.40^{\mathrm{c}}$ & $0.12^{\mathrm{b}}$ & $39.41^{\mathrm{cB}}$ \\
\hline 13 & & 0.01 & 0.00 & 0.00 & 0. & 0.00 & 0. & 0. & 0 . & 0.00 & 0. & 0.00 & 0.21 \\
\hline & & $0.24^{\mathrm{a}}$ & 0.06 & $0.03^{\mathrm{a}}$ & $11.58^{\mathrm{ab}}$ & $0.63^{\mathrm{a}}$ & $21.78^{b c}$ & $0.86^{\mathrm{a}}$ & $6.09^{b}$ & $0.40^{\mathrm{a}}$ & $0.52^{\mathrm{b}}$ & $0.11^{\mathrm{c}}$ & $42.27^{\mathrm{bAB}}$ \\
\hline & 0.8 & 0. & 0.00 & 0.00 & & 0. & & & 0 . & 0.04 & 0. & 0 & 0.16 \\
\hline & & $0.08^{c}$ & 0.05 & DD & $11.20^{\mathrm{b}}$ & $0.63^{\mathrm{a}}$ & $21.84^{\mathrm{b}}$ & $0.87^{\mathrm{a}}$ & $5.89^{\mathrm{b}}$ & $0.61^{\mathrm{a}}$ & $0.51^{\mathrm{b}}$ & $0.07^{\mathrm{d}}$ & $41.73^{\mathrm{bAB}}$ \\
\hline & $0.8 \mathrm{P}$ & 0.00 & 0.00 & ND & 0.96 & 0.01 & 0.4 & 0.1 & 0.16 & 0.30 & 0.00 & 0.01 & 0.78 \\
\hline & C & $0.25^{\mathrm{ab}}$ & $0.07^{\mathrm{b}}$ & & $12.16^{\mathrm{a}}$ & $0.71^{\mathrm{a}}$ & $21.25^{\mathrm{a}}$ & $0.91^{\mathrm{a}}$ & $5.45^{\mathrm{b}}$ & $0.37^{\mathrm{c}}$ & $0.53^{\mathrm{b}}$ & $0.16^{\mathrm{a}}$ & $41.83^{\mathrm{aA}}$ \\
\hline & C & 0.08 & 0.01 & & & 0.01 & & 0.0 & 0.25 & 0.01 & 0.01 & 0.01 & 0.18 \\
\hline & & $0.16^{\mathrm{c}}$ & $0.08^{\mathrm{a}}$ & & $10.5^{\mathrm{b}}$ & $0.60^{\mathrm{b}}$ & $21.98^{\mathrm{a}}$ & $0.79^{\mathrm{a}}$ & $6.32^{\mathrm{a}}$ & $0.56^{\mathrm{a}}$ & $0.52^{\mathrm{b}}$ & $0.09^{\mathrm{a}}$ & $41.57^{\mathrm{aB}}$ \\
\hline & & 0.01 & 0.00 & & 0.71 & 0.04 & 0.66 & 0.08 & 0.52 & 0.04 & 0.02 & 0.01 & 0.37 \\
\hline 1 & & $0.27^{\mathrm{a}}$ & $0.04^{\mathrm{c}}$ & & $11.84^{\mathrm{a}}$ & $0.61^{b}$ & $20.45^{\mathrm{a}}$ & $0.89^{\mathrm{a}}$ & $5.58^{\mathrm{ab}}$ & $0.34^{c}$ & $0.57^{\mathrm{a}}$ & $0.16^{\mathrm{a}}$ & $40.72^{\mathrm{aAB}}$ \\
\hline $1 J$ & & 0.00 & 0.00 & & 0.37 & 0.00 & 1.38 & 0.03 & 0.22 & 0.01 & 0.01 & 0.01 & 1.20 \\
\hline & & $0.24^{\mathrm{ab}}$ & $0.08^{\mathrm{a}}$ & 0.03 & $11.16^{\mathrm{ab}}$ & $0.61^{b}$ & $21.29^{\mathrm{a}}$ & $0.82^{\mathrm{a}}$ & $6.10^{\mathrm{ab}}$ & $0.50^{\mathrm{b}}$ & $0.53^{\mathrm{b}}$ & $0.18^{\mathrm{a}}$ & $41.50^{\mathrm{aB}}$ \\
\hline & & 0.01 & 0.01 & 0.00 & 0.37 & 0.04 & 0.30 & 0.04 & 0.01 & 0.01 & 0.01 & 0.01 & 0.75 \\
\hline & & $0.17^{\mathrm{c}}$ & $0.07^{\mathrm{b}}$ & & $10.98^{\mathrm{ab}}$ & $0.60^{\mathrm{b}}$ & $21.26^{\mathrm{a}}$ & $0.80^{\mathrm{a}}$ & $6.03^{\mathrm{ab}}$ & $0.61^{\mathrm{a}}$ & $0.53^{\mathrm{b}}$ & $0.16^{\mathrm{a}}$ & $41.16^{\mathrm{a} A \mathrm{~B}}$ \\
\hline & 0.8 & 0.01 & 0.01 & ND & 0.15 & 0.01 & 0.21 & 0.01 & 0.15 & 0.01 & 0.01 & 0.02 & 0.24 \\
\hline
\end{tabular}

${ }^{\mathrm{x}}$ Average value, ${ }^{\mathrm{y}}$ Standard deviation $(\mathrm{n}=3)$. ND: not detected. Means followed by different letters are significantly different $(\mathrm{P}<0.05)$ among groups $\left({ }^{\mathrm{a}-\mathrm{c}}\right)$ and during storage ${ }^{(\mathrm{A}-\mathrm{C})} \mathrm{C}$ : Control group, $0.4 \mathrm{PE}$ : group treated with ethanolic extract of propolis at doses of $0.4 \%$, $0.8 \mathrm{PE}$ : group treated with ethanolic extract of propolis at doses of $0.8 \%, 0.4 \mathrm{PW}$ : group treated with water extract of propolis at doses of $0.4 \%, 0.4 \mathrm{PW}$ : group treated with water extract of propolis at doses of $0.8 \%$. 
Table 2. Changes in MUFA (\%) content of sardine treated with/without water or ethanolic extract of propolis during chill storage

\begin{tabular}{|c|c|c|c|c|c|c|c|c|c|c|c|}
\hline Days & Groups & C14:1 & C15:1 & C16:1 & C17:1 & C18:1n9 & $\mathrm{C} 18: 1 \mathrm{n} 7$ & C20:1n9 & $\mathrm{C} 22: \ln 9$ & $\mathrm{C} 24: \ln 9$ & MUFA \\
\hline \multirow{2}{*}{0} & \multirow{2}{*}{ C } & $0.19^{\mathrm{x}}$ & 0.02 & 14.05 & 0.8 & 4.88 & 4.07 & 0.17 & \multirow{2}{*}{ ND } & 0.23 & $24.37^{\mathrm{AB}}$ \\
\hline & & $0.01^{\mathrm{y}}$ & 0.00 & 0.23 & 0.01 & 0.42 & 0.29 & 0.01 & & 0.28 & 0.76 \\
\hline \multirow{10}{*}{4} & \multirow{2}{*}{ C } & $0.18^{\mathrm{a}}$ & $0.01^{\mathrm{a}}$ & $13.83^{\mathrm{a}}$ & $0.65^{\mathrm{bc}}$ & $4.46^{\mathrm{c}}$ & $4.615^{\mathrm{a}}$ & $0.165^{\mathrm{a}}$ & $0.015^{\mathrm{b}}$ & $0.22^{\mathrm{a}}$ & $24.17^{\mathrm{aAB}}$ \\
\hline & & 0.02 & 0.00 & 0.69 & 0.07 & 0.01 & 0.36 & 0.04 & 0.02 & 0.29 & 0.11 \\
\hline & \multirow{2}{*}{$0.4 \mathrm{PE}$} & $0.17^{\mathrm{a}}$ & $0.04^{\mathrm{a}}$ & $12.81^{\mathrm{b}}$ & $0.59^{c}$ & $5.91^{\mathrm{a}}$ & $4.44^{\mathrm{a}}$ & $0.14^{\mathrm{a}}$ & $0.32^{\mathrm{a}}$ & $0.04^{\mathrm{a}}$ & $24.48^{\mathrm{aA}}$ \\
\hline & & 0.00 & 0.00 & 0.29 & 0.04 & 0.01 & 0.01 & 0.00 & 0.02 & 0.01 & 0.37 \\
\hline & \multirow{2}{*}{$0.4 \mathrm{PW}$} & $0.11^{\mathrm{b}}$ & $0.03^{\mathrm{a}}$ & $14.18^{\mathrm{a}}$ & $0.78^{\mathrm{a}}$ & $4.65^{b c}$ & $4.38^{\mathrm{a}}$ & $0.15^{\mathrm{a}}$ & $0.04^{\mathrm{b}}$ & $0.06^{\mathrm{a}}$ & $24.37^{\mathrm{aAB}}$ \\
\hline & & 0.01 & 0.00 & 0.02 & 0.00 & 0.17 & 0.04 & 0.04 & 0.00 & 0.00 & 0.13 \\
\hline & \multirow{2}{*}{$0.8 \mathrm{PE}$} & $0.07^{c}$ & $0.04^{\mathrm{a}}$ & $13.9^{\mathrm{a}}$ & $0.69^{\mathrm{abc}}$ & $5.10^{\mathrm{b}}$ & $4.55^{\mathrm{a}}$ & $0.15^{\mathrm{a}}$ & $0.04^{\mathrm{b}}$ & $0.06^{\mathrm{a}}$ & $24.58^{\mathrm{aA}}$ \\
\hline & & 0.00 & 0.01 & 0.12 & 0.02 & 0.4 & 0.1 & 0.01 & 0.00 & 0.00 & 0.4 \\
\hline & \multirow{2}{*}{$0.8 \mathrm{PW}$} & $0.10^{\mathrm{b}}$ & $0.06^{\mathrm{a}}$ & $13.4^{\mathrm{ab}}$ & $0.71^{\mathrm{ab}}$ & $4.98^{\mathrm{bc}}$ & 4.26 & $0.14^{\mathrm{a}}$ & $0.05^{\mathrm{b}}$ & $0.05^{\mathrm{a}}$ & $23.73^{\mathrm{aB}}$ \\
\hline & & 0.00 & 0.04 & 0.23 & 0.00 & 0.29 & 0.11 & 0.01 & 0.00 & 0.00 & 0.61 \\
\hline \multirow{10}{*}{7} & \multirow{2}{*}{ C } & $0.03^{c}$ & $0.04^{\mathrm{ab}}$ & $14.1^{\mathrm{a}}$ & $0.82^{\mathrm{a}}$ & $4.43^{\mathrm{b}}$ & $4.18^{\mathrm{b}}$ & $0.15^{\mathrm{a}}$ & $0.02^{\mathrm{b}}$ & $0.04^{\mathrm{b}}$ & $23.79^{\mathrm{bcB}}$ \\
\hline & & 0.00 & 0.01 & 0.03 & 0.06 & 0.00 & 0.14 & 0.01 & 0.00 & 0.01 & 0.05 \\
\hline & \multirow{2}{*}{$0.4 \mathrm{PE}$} & $0.03^{c}$ & $0.04^{\mathrm{ab}}$ & $14.06^{\mathrm{a}}$ & $0.66^{\mathrm{a}}$ & $4.70^{\mathrm{ab}}$ & $4.69^{\mathrm{a}}$ & $0.15^{\mathrm{a}}$ & $0.05^{\mathrm{a}}$ & $0.06^{\mathrm{a}}$ & $24.41^{\mathrm{abA}}$ \\
\hline & & 0.00 & 0.00 & 0.15 & 0.04 & 0.27 & 0.33 & 0.01 & 0.01 & 0.01 & 0.51 \\
\hline & 0 4PW & $0.03^{c}$ & $0.03^{\mathrm{b}}$ & $14.10^{\mathrm{a}}$ & $0.83^{\mathrm{a}}$ & $5.05^{\mathrm{a}}$ & $4.62^{\mathrm{ab}}$ & $0.14^{\mathrm{a}}$ & $0.05^{\mathrm{a}}$ & $0.07^{\mathrm{a}}$ & $24.91^{\mathrm{aA}}$ \\
\hline & $0.4 \mathrm{~T} \mathrm{~W}$ & 0.00 & 0.00 & 0.47 & 0.18 & 0.13 & 0.13 & 0.01 & 0.00 & 0.01 & 0.02 \\
\hline & O \&PF & $0.10^{\mathrm{a}}$ & $0.05^{\mathrm{a}}$ & $14.13^{\mathrm{a}}$ & $0.89^{\mathrm{a}}$ & $4.32^{\mathrm{b}}$ & $4.28^{\mathrm{ab}}$ & $0.16^{\mathrm{a}}$ & $0.03^{\mathrm{b}}$ & $0.06^{\mathrm{a}}$ & $23.99^{\mathrm{bcA}}$ \\
\hline & $0.0 \mathrm{~F} \mathrm{E}$ & 0.01 & 0.01 & 0.45 & 0.13 & 0.26 & 0.04 & 0.01 & 0.01 & 0.01 & 0.08 \\
\hline & 0 8PW & $0.07^{\mathrm{b}}$ & $0.03^{\mathrm{b}}$ & $13.33^{\mathrm{a}}$ & $0.73^{\mathrm{a}}$ & $4.87^{\mathrm{ab}}$ & $4.24^{\mathrm{ab}}$ & $0.16^{\mathrm{a}}$ & $0.03^{\mathrm{b}}$ & $0.04^{\mathrm{b}}$ & $23.48^{\mathrm{cBC}}$ \\
\hline & $0.01 \mathrm{~W}$ & 0.00 & 0.01 & 0.05 & 0.1 & 0.3 & 0.15 & 0.00 & 0.00 & 0.00 & 0.93 \\
\hline & $\mathrm{C}$ & $0.07^{\mathrm{a}}$ & $0.04^{\mathrm{a}}$ & $13.92^{\mathrm{ab}}$ & $0.67^{\mathrm{a}}$ & $4.87^{\mathrm{a}}$ & $4.85^{\mathrm{a}}$ & $0.20^{\mathrm{a}}$ & ND & $0.04^{\mathrm{b}}$ & $24.63^{\mathrm{abAB}}$ \\
\hline & $c$ & 0.01 & 0.01 & 0.14 & 0.02 & 0.33 & 0.01 & 0.01 & ND & 0.01 & 0.18 \\
\hline & $04 \mathrm{PF}$ & $0.07^{\mathrm{a}}$ & ND & $14.06^{\mathrm{ab}}$ & $0.64^{\mathrm{a}}$ & $5.14^{\mathrm{a}}$ & $4.68^{\mathrm{a}}$ & $0.15^{c}$ & ND & $0.05^{\mathrm{a}}$ & $24.77^{\mathrm{abA}}$ \\
\hline & $0.4 \mathrm{PE}$ & 0.01 & ND & 0.25 & 0.08 & 0.28 & 0.3 & 0.00 & ND & 0.00 & 0.24 \\
\hline 9 & $04 \mathrm{PW}$ & $0.06^{\mathrm{ab}}$ & $0.04^{\mathrm{a}}$ & $12.78^{\mathrm{b}}$ & $0.63^{\mathrm{a}}$ & $5.56^{\mathrm{a}}$ & $4.30^{\mathrm{a}}$ & $0.16^{\mathrm{bc}}$ & 0.02 & $0.04^{\mathrm{b}}$ & $23.58^{\mathrm{bB}}$ \\
\hline 9 & $0.4 \mathrm{PW}$ & 0.00 & 0.00 & 0.3 & 0.05 & 0.2 & 0.22 & 0.00 & 0.00 & 0.00 & 0.52 \\
\hline & 0 8PE & $0.07^{\mathrm{a}}$ & $0.03^{\mathrm{a}}$ & $13.70^{\mathrm{ab}}$ & $0.61^{\mathrm{a}}$ & $5.23^{\mathrm{a}}$ & $4.4 \mathrm{a}$ & $0.17^{\mathrm{b}}$ & ND & $0.02^{\mathrm{b}}$ & $24.22^{\mathrm{abA}}$ \\
\hline & $0.8 \mathrm{FE}$ & 0.00 & 0.00 & 0.99 & 0.01 & 0.32 & 0.01 & 0.01 & ND & 0.00 & 1.86 \\
\hline & & $0.06^{\mathrm{ab}}$ & $0.05^{\mathrm{a}}$ & $14.31^{\mathrm{a}}$ & $0.63^{\mathrm{a}}$ & $5.07^{\mathrm{a}}$ & $4.53^{\mathrm{a}}$ & $0.19^{\mathrm{a}}$ & ND & $0.46^{\mathrm{b}}$ & $25.28^{\mathrm{aA}}$ \\
\hline & $0.8 \mathrm{PW}$ & 0.01 & 0.00 & 0.14 & 0.07 & 0.4 & 0.26 & 0.01 & ND & 0.05 & 0.38 \\
\hline & $\mathrm{C}$ & $0.07^{\mathrm{a}}$ & 0.04 & $14.33^{\mathrm{a}}$ & $0.76^{\mathrm{a}}$ & $5.09^{\mathrm{ab}}$ & $4.01^{\mathrm{a}}$ & $0.19^{\mathrm{a}}$ & $0.03^{\mathrm{a}}$ & $0.05^{\mathrm{ab}}$ & $24.56^{\mathrm{aAB}}$ \\
\hline & $c$ & 0.00 & 0.00 & 0.12 & 0.00 & 0.35 & 0.8 & 0.00 & 0.00 & 0.00 & 0.57 \\
\hline & $04 \mathrm{~F}$ & $0.06^{\mathrm{b}}$ & $0.04^{\mathrm{a}}$ & $13.50^{\mathrm{ab}}$ & $0.69^{\mathrm{a}}$ & $5.02^{\mathrm{ab}}$ & $4.54^{\mathrm{a}}$ & $0.14^{\mathrm{a}}$ & $0.02^{\mathrm{a}}$ & $0.04^{b}$ & $24.03^{\mathrm{aA}}$ \\
\hline & $0.4 \mathrm{E}$ & 0.00 & 0.01 & 0.13 & 0.02 & 0.16 & 0.29 & 0.00 & 0.00 & 0.00 & 0.57 \\
\hline 11 & $04 \mathrm{PW}$ & $0.07^{\mathrm{a}}$ & $0.04^{\mathrm{a}}$ & $13.73^{\mathrm{ab}}$ & $0.75^{\mathrm{a}}$ & $4.61^{\mathrm{b}}$ & $4.26^{\mathrm{a}}$ & $0.16^{\mathrm{a}}$ & $0.03^{\mathrm{a}}$ & $0.06^{\mathrm{a}}$ & $23.70^{\mathrm{abB}}$ \\
\hline 11 & $0.41 \mathrm{~W}$ & 0.00 & 0.00 & 0.54 & 0.08 & 0.02 & 0.06 & 0.03 & 0.00 & 0.01 & 0.57 \\
\hline & 0 8PF & $0.07^{\mathrm{a}}$ & $0.03^{b}$ & $13.89^{\mathrm{a}}$ & $0.74^{\mathrm{a}}$ & $4.7^{\mathrm{ab}}$ & $4.42^{\mathrm{a}}$ & $0.14^{\mathrm{a}}$ & $0.02^{\mathrm{a}}$ & $0.06^{\mathrm{a}}$ & $24.06^{\mathrm{aA}}$ \\
\hline & $0.8 \mathrm{PE}$ & 0.00 & 0.00 & 0.41 & 0.00 & 0.01 & 0.18 & 0.01 & 0.00 & 0.01 & 0.56 \\
\hline & 0 8PW & $0.07^{\mathrm{a}}$ & $0.04^{\mathrm{a}}$ & $12.69^{b}$ & $0.58^{\mathrm{b}}$ & $5.06^{\mathrm{a}}$ & $3.86^{\mathrm{a}}$ & $0.15^{\mathrm{a}}$ & $0.00^{\mathrm{a}}$ & $0.04^{\mathrm{b}}$ & $22.48^{\mathrm{bC}}$ \\
\hline & $0.01 \mathrm{vv}$ & 0.01 & 0.00 & 0.71 & 0.04 & 0.00 & 0.14 & 0.00 & 0.00 & 0.00 & 0.52 \\
\hline & $\mathrm{C}$ & $0.07^{\mathrm{a}}$ & $0.04^{\mathrm{ab}}$ & $14.76^{\mathrm{a}}$ & $0.74^{\mathrm{a}}$ & $4.75^{\mathrm{a}}$ & $4.58^{\mathrm{a}}$ & $0.15^{\mathrm{a}}$ & $0.00^{\mathrm{a}}$ & $0.03^{c}$ & $25.11^{\mathrm{aA}}$ \\
\hline & & 0.00 & 0.01 & 0.93 & 0.01 & 0.36 & 0.23 & 0.01 & 0.00 & 0.00 & 0.31 \\
\hline & $04 \mathrm{PF}$ & $0.06^{\mathrm{a}}$ & $0.04^{\mathrm{ab}}$ & $12.97^{\mathrm{c}}$ & $0.68^{\mathrm{a}}$ & $4.85^{\mathrm{a}}$ & $4.40^{\mathrm{a}}$ & $0.16^{\mathrm{a}}$ & $0.01^{\mathrm{a}}$ & $0.01^{\mathrm{d}}$ & $23.17^{\mathrm{bA}}$ \\
\hline & $0.4 \mathrm{~T}$ & 0.00 & 0.00 & 0.88 & 0.03 & 0.27 & 0.13 & 0.00 & 0.00 & 0.00 & 1.46 \\
\hline 13 & $04 \mathrm{PW}$ & $0.07^{\mathrm{a}}$ & $0.03^{\mathrm{b}}$ & $14.59^{\mathrm{a}}$ & $0.40^{\mathrm{a}}$ & $4.58^{\mathrm{a}}$ & $4.60^{\mathrm{a}}$ & $0.14^{\mathrm{a}}$ & $0.02^{\mathrm{a}}$ & $0.04^{\mathrm{b}}$ & $24.45^{\mathrm{abAB}}$ \\
\hline 13 & $0.4 \mathrm{~F}$ & 0.01 & 0.00 & 0.06 & 0.01 & 0.17 & 0.04 & 0.01 & 0.00 & 0.00 & 0.65 \\
\hline & 08 & $0.06^{\mathrm{a}}$ & $0.05^{\mathrm{a}}$ & $13.29^{\mathrm{ab}}$ & $0.64^{\mathrm{a}}$ & $4.92^{\mathrm{a}}$ & $4.40^{\mathrm{a}}$ & $0.15^{\mathrm{a}}$ & $0.01^{\mathrm{a}}$ & $0.05^{\mathrm{b}}$ & $23.56^{\mathrm{abA}}$ \\
\hline & 0.0 & 0.00 & 0.01 & 0.42 & 0.04 & 0.31 & 0.01 & 0.01 & 0.00 & 0.01 & 0.12 \\
\hline & & $0.06^{\mathrm{a}}$ & $0.03^{b}$ & $13.05^{\mathrm{bc}}$ & $0.71^{\mathrm{a}}$ & $5.16^{\mathrm{a}}$ & $4.19^{\mathrm{a}}$ & $0.14^{\mathrm{a}}$ & $0.07^{\mathrm{a}}$ & $0.24^{\mathrm{a}}$ & $23.63^{\mathrm{abB}}$ \\
\hline & $0.8 \mathrm{PW}$ & 0.00 & 0.00 & 0.02 & 0.18 & 0.34 & 0.33 & 0.02 & 0.08 & 0.00 & 0.08 \\
\hline & C & $0.08^{a}$ & $0.03^{\mathrm{a}}$ & $13.86^{\mathrm{a}}$ & $0.73^{\mathrm{a}}$ & $4.55^{\mathrm{b}}$ & $4.18^{\mathrm{b}}$ & $0.19^{\mathrm{a}}$ & ND & $0.43^{\mathrm{a}}$ & $24.04^{\mathrm{aAB}}$ \\
\hline & & 0.00 & 0.00 & 0.48 & 0.08 & 0.09 & 0.07 & 0.03 & & 0.01 & 0.57 \\
\hline & १ 4PF & $0.06^{\mathrm{a}}$ & $0.04^{\mathrm{a}}$ & $12.37^{b}$ & $0.54^{\mathrm{b}}$ & $6.64^{\mathrm{a}}$ & $4.50^{\mathrm{ab}}$ & $0.18^{\mathrm{a}}$ & $N D$ & $0.24^{\mathrm{b}}$ & $24.55^{\mathrm{aA}}$ \\
\hline & $0.4 \mathrm{~F} \mathrm{E}$ & 0.00 & 0.00 & 0.73 & 0.06 & 0.01 & 0.04 & 0.01 & ND & 0.01 & 0.76 \\
\hline 15 & $04 \mathrm{PW}$ & $0.07^{\mathrm{a}}$ & $0.04^{\mathrm{a}}$ & $13.73^{\mathrm{a}}$ & $0.64^{\mathrm{ab}}$ & $4.94^{\mathrm{b}}$ & $4.89^{\mathrm{a}}$ & $0.17^{\mathrm{a}}$ & ND & $0.03^{\mathrm{c}}$ & $24.49^{\mathrm{aAB}}$ \\
\hline $1 J$ & $0.4 \mathrm{~F} \mathrm{~V}$ & 0.00 & 0.00 & 0.35 & 0.04 & 0.35 & 0.06 & 0.01 & ND & 0.00 & 0.02 \\
\hline & 0 8PF & $0.07^{\mathrm{a}}$ & $0.04^{\mathrm{a}}$ & $12.67^{\mathrm{ab}}$ & $0.59^{\mathrm{b}}$ & $6.54^{\mathrm{a}}$ & $4.56^{\mathrm{ab}}$ & $0.17^{\mathrm{a}}$ & ND & $0.02^{\mathrm{c}}$ & $24.65^{\mathrm{aA}}$ \\
\hline & $0.8 \mathrm{PE}$ & 0.00 & 0.00 & 0.47 & 0.05 & 0.49 & 0.37 & 0.00 & ND & 0.00 & 0.77 \\
\hline & 0 8PW & $0.07^{\mathrm{a}}$ & $0.05^{\mathrm{a}}$ & $12.58^{\mathrm{ab}}$ & $0.57^{b}$ & $6.09^{\mathrm{a}}$ & $4.46^{\mathrm{ab}}$ & $0.16^{\mathrm{a}}$ & ND & $0.23^{\mathrm{b}}$ & $24.18^{\mathrm{aAB}}$ \\
\hline & $0.8 \mathrm{PW}$ & 0.00 & 0.00 & 0.19 & 0.01 & 0.48 & 0.02 & 0.01 & ND & 0.01 & 0.69 \\
\hline
\end{tabular}

${ }^{\mathrm{x}}$ Average value, ${ }^{\mathrm{y} S t a n d a r d ~ d e v i a t i o n}(\mathrm{n}=3)$. ND: not detected. Means followed by different letters are significantly different $(\mathrm{P}<0.05)$ among groups $\left({ }^{\mathrm{a}-\mathrm{C}}\right)$ and during storage ${ }^{(\mathrm{A}-\mathrm{C})}$. C: Control group, $0.4 \mathrm{PE}$ : group treated with ethanolic extract of propolis at doses of $0.4 \%, 0.8 \mathrm{PE}$ : group treated with ethanolic extract of propolis at doses of $0.8 \%, 0.4 \mathrm{PW}$ : group treated with water extract of propolis at doses of $0.4 \%, 0.4 \mathrm{PW}$ : group treated with water extract of propolis at doses of $0.8 \%$. 
Table 3. Changes in PUFA (\%) content of sardine treated with/without water or ethanolic extract of propolis during chill storage

\begin{tabular}{|c|c|c|c|c|c|c|c|c|c|c|}
\hline Days & Groups & C18:2n6 & C18:3n6 & $\mathrm{C} 18: 3 \mathrm{n} 3$ & C20:2cis & $\mathrm{C} 20: 4 \mathrm{n} 6$ & $\mathrm{C} 20: 5 \mathrm{n} 3$ & C22:2cis & $\mathrm{C} 22: 6 \mathrm{n} 3$ & PUFA \\
\hline \multirow{2}{*}{0} & \multirow{2}{*}{$\mathrm{C}$} & $2.03^{x}$ & 0.08 & 0.24 & 0.16 & 0.96 & 10.95 & 0.02 & 7.16 & $21.59^{\mathrm{B}}$ \\
\hline & & $0.24^{\mathrm{y}}$ & 0.00 & 0.01 & 0.01 & 0.07 & 0.4 & 0.00 & 0.66 & 0.74 \\
\hline \multirow{10}{*}{4} & \multirow{2}{*}{$\mathrm{C}$} & $1.90^{\mathrm{ab}}$ & $0.40^{\mathrm{a}}$ & $0.26^{\mathrm{a}}$ & $0.19^{\mathrm{a}}$ & $0.57^{\mathrm{b}}$ & $10.11^{\mathrm{b}}$ & $0.07^{\mathrm{a}}$ & $6.21^{\mathrm{c}}$ & $19.73^{\mathrm{bC}}$ \\
\hline & & 0.08 & 0.03 & 0.03 & 0.01 & 0.04 & 0.91 & 0.01 & 0.33 & 1.06 \\
\hline & \multirow{2}{*}{$0.4 \mathrm{PE}$} & $1.87^{\mathrm{bc}}$ & $0.08^{\mathrm{b}}$ & $0.03^{\mathrm{b}}$ & $0.16^{\mathrm{b}}$ & $0.53^{\mathrm{bc}}$ & $11.57^{\mathrm{ab}}$ & $0.09^{\mathrm{a}}$ & $8.18^{\mathrm{a}}$ & $22.54^{\mathrm{aA}}$ \\
\hline & & 0.06 & 0.00 & 0.01 & 0.01 & 0.02 & 0.91 & 0.00 & 0.22 & 0.66 \\
\hline & \multirow{2}{*}{$0.4 \mathrm{PW}$} & $1.77^{\mathrm{c}}$ & $0.09^{\mathrm{b}}$ & $0.04^{\mathrm{b}}$ & $0.16^{\mathrm{b}}$ & $1.07^{\mathrm{a}}$ & $12.22^{\mathrm{a}}$ & $0.05^{\mathrm{b}}$ & $7.49^{\mathrm{ab}}$ & $22.87^{\mathrm{aA}}$ \\
\hline & & 0.01 & 0.00 & 0.00 & 0.01 & 0.02 & 0.00 & 0.01 & 0.11 & 0.08 \\
\hline & \multirow{2}{*}{$0.8 \mathrm{PE}$} & $2.01^{\mathrm{a}}$ & $0.10^{\mathrm{b}}$ & $0.05^{\mathrm{b}}$ & $0.16^{\mathrm{b}}$ & $1.11^{\mathrm{a}}$ & $11.84^{\mathrm{ab}}$ & $0.04^{\mathrm{b}}$ & $7.34^{\mathrm{b}}$ & $22.64^{\mathrm{aA}}$ \\
\hline & & 0.01 & 0.00 & 0.00 & 0.01 & 0.06 & 0.99 & 0.00 & 0.11 & 1.03 \\
\hline & \multirow{2}{*}{$0.8 \mathrm{PW}$} & $1.91^{\mathrm{ab}}$ & $0.09^{\mathrm{b}}$ & $0.04^{\mathrm{b}}$ & $0.17^{\mathrm{b}}$ & $0.48^{c}$ & $10.35^{\mathrm{ab}}$ & $0.10^{\mathrm{a}}$ & $8.03^{\mathrm{a}}$ & $21.16^{\mathrm{abA}}$ \\
\hline & & 0.04 & 0.01 & 0.00 & 0.00 & 0.04 & 0.27 & 0.01 & 0.54 & 0.74 \\
\hline \multirow{10}{*}{7} & \multirow{2}{*}{$\mathrm{C}$} & $1.01^{\mathrm{b}}$ & $0.08^{\mathrm{a}}$ & $0.03^{\mathrm{a}}$ & $0.18^{\mathrm{a}}$ & $0.03^{\mathrm{d}}$ & $11.00^{\mathrm{ab}}$ & $0.14^{\mathrm{a}}$ & $7.59^{\mathrm{abc}}$ & $20.05^{\mathrm{bBC}}$ \\
\hline & & 0.01 & 0.00 & 0.00 & 0.01 & 0.00 & 0.48 & 0.01 & 0.16 & 0.31 \\
\hline & \multirow{2}{*}{$0.4 \mathrm{PE}$} & $1.09^{\mathrm{b}}$ & $0.09^{\mathrm{a}}$ & $0.03^{\mathrm{a}}$ & $0.17^{\mathrm{a}}$ & $0.64^{\mathrm{b}}$ & $11.44^{\mathrm{ab}}$ & $0.15^{\mathrm{a}}$ & $7.23^{\mathrm{bc}}$ & $20.78^{\mathrm{abA}}$ \\
\hline & & 0.06 & 0.00 & 0.00 & 0.01 & 0.04 & 0.40 & 0.04 & 0.7 & 1.94 \\
\hline & \multirow{2}{*}{$0.4 \mathrm{PW}$} & $1.65^{\mathrm{a}}$ & $0.09^{\mathrm{a}}$ & $0.03^{\mathrm{a}}$ & $0.16^{\mathrm{a}}$ & $0.02^{\mathrm{d}}$ & $12.15^{\mathrm{a}}$ & $0.07^{\mathrm{ab}}$ & $6.77^{\mathrm{c}}$ & $20.92^{\mathrm{abC}}$ \\
\hline & & 0.08 & 0.01 & 0.00 & 0.01 & 0.00 & 0.22 & 0.01 & 0.01 & 0.3 \\
\hline & $08 \mathrm{PF}$ & $1.69^{\mathrm{a}}$ & $0.10^{\mathrm{a}}$ & $0.04^{\mathrm{a}}$ & $0.18^{\mathrm{a}}$ & $0.75^{\mathrm{a}}$ & $11.15^{\mathrm{ab}}$ & $0.05^{\mathrm{ab}}$ & $8.20^{\mathrm{ab}}$ & $22.14^{\mathrm{aAB}}$ \\
\hline & $0.01 \mathrm{E}$ & 0.08 & 0.01 & 0.00 & 0.01 & 0.01 & 0.84 & 0.00 & 0.23 & 0.64 \\
\hline & Q 8PW & $1.03^{\mathrm{b}}$ & $0.08^{\mathrm{a}}$ & $0.03^{\mathrm{a}}$ & $0.19^{\mathrm{a}}$ & $0.39^{c}$ & $10.79^{\mathrm{b}}$ & $0.15^{\mathrm{a}}$ & $8.47^{\mathrm{a}}$ & $21.12^{\mathrm{bA}}$ \\
\hline & $0.8 \mathrm{PW}$ & 0.01 & 0.01 & 0.00 & 0.02 & 0.03 & 0.33 & 0.01 & 0.5 & 1.8 \\
\hline & $C$ & $1.92^{\mathrm{b}}$ & $0.08^{\mathrm{a}}$ & $0.03^{\mathrm{c}}$ & $0.19^{\mathrm{b}}$ & $1.05^{\mathrm{b}}$ & $10.87^{\mathrm{ab}}$ & $0.05^{\mathrm{ab}}$ & $7.03^{b}$ & $21.19^{\mathrm{cBC}}$ \\
\hline & $c$ & 0.15 & 0.01 & 0.00 & 0.02 & 0.05 & 0.06 & 0.00 & 0.30 & 0.16 \\
\hline & $\Omega \triangle \mathrm{PE}$ & $1.90^{\mathrm{b}}$ & $0.08^{\mathrm{a}}$ & $0.39^{\mathrm{a}}$ & $0.17^{\mathrm{b}}$ & $1.24^{\mathrm{a}}$ & $11.70^{\mathrm{a}}$ & $0.04^{\mathrm{b}}$ & $7.30^{\mathrm{b}}$ & $22.78^{\mathrm{bA}}$ \\
\hline & $0.41 \mathrm{~L}$ & 0.04 & 0.00 & 0.02 & 0.00 & 0.01 & 0.52 & 0.00 & 0.22 & 0.78 \\
\hline 9 & 0 4PW & $2.67^{\mathrm{a}}$ & $0.07^{\mathrm{a}}$ & $0.02^{\mathrm{c}}$ & $0.18^{\mathrm{ab}}$ & $1.12^{\mathrm{b}}$ & $10.67^{\mathrm{b}}$ & $0.09^{a}$ & $8.31^{\mathrm{a}}$ & $22.46^{\mathrm{bAB}}$ \\
\hline 9 & $0.4 \mathrm{~F} \mathrm{~V}$ & 0.17 & 0.00 & 0.00 & 0.01 & 0.01 & 0.49 & 0.01 & 0.05 & 0.28 \\
\hline & $08 \mathrm{PF}$ & $2.16^{\mathrm{ab}}$ & $0.07^{\mathrm{a}}$ & $0.02^{\mathrm{c}}$ & $0.22^{\mathrm{a}}$ & $1.09^{\mathrm{b}}$ & $11.53^{\mathrm{ab}}$ & $0.06^{\mathrm{a}}$ & $8.07^{\mathrm{ab}}$ & $25.66^{\mathrm{aC}}$ \\
\hline & $0.8 \mathrm{PE}$ & 0.03 & 0.00 & 0.00 & 0.02 & 0.08 & 0.18 & 0.00 & 0.4 & 0.35 \\
\hline & 0 8PW & $1.84^{\mathrm{a}}$ & $0.09^{\mathrm{a}}$ & $0.15^{\mathrm{b}}$ & $0.17^{\mathrm{b}}$ & $1.15^{\mathrm{ab}}$ & $11.60^{\mathrm{a}}$ & $0.05^{\mathrm{ab}}$ & $7.34^{\mathrm{b}}$ & $22.36^{\mathrm{bA}}$ \\
\hline & $0.8 \mathrm{PW}$ & 0.01 & 0.02 & 0.01 & 0.01 & 0.01 & 0.27 & 0.00 & 0.35 & 0.78 \\
\hline & & $1.90^{\mathrm{b}}$ & $0.09^{\mathrm{a}}$ & $0.03^{\mathrm{b}}$ & $0.17^{\mathrm{b}}$ & $1.02^{\mathrm{a}}$ & $11.87^{\mathrm{a}}$ & $0.04^{\mathrm{c}}$ & $7.81^{\mathrm{a}}$ & $22.93^{\mathrm{aA}}$ \\
\hline & $\mathrm{C}$ & 0.00 & 0.00 & 0.00 & 0.00 & 0.00 & 0.43 & 0.00 & 0.68 & 0.25 \\
\hline & $04 \mathrm{PF}$ & $2.02^{\mathrm{b}}$ & $0.07^{\mathrm{a}}$ & $0.05^{\mathrm{b}}$ & $0.19^{\mathrm{ab}}$ & $0.09^{\mathrm{d}}$ & $11.32^{\mathrm{a}}$ & $0.17^{\mathrm{ab}}$ & $7.87^{\mathrm{a}}$ & $21.75^{\mathrm{aA}}$ \\
\hline & $0.4 \mathrm{PE}$ & 0.04 & 0.05 & 0.02 & 0.01 & 0.00 & 0.08 & 0.01 & 0.28 & 0.24 \\
\hline 11 & $04 \mathrm{PW}$ & $1.93^{\mathrm{b}}$ & $0.09^{\mathrm{a}}$ & $0.04^{\mathrm{b}}$ & $0.19^{\mathrm{ab}}$ & $0.95^{\mathrm{b}}$ & $10.90^{\mathrm{a}}$ & $0.10^{\mathrm{abc}}$ & $8.30^{\mathrm{a}}$ & $22.48^{\mathrm{aAB}}$ \\
\hline 11 & $0.4 \mathrm{PW}$ & 0.04 & 0.01 & 0.01 & 0.01 & 0.01 & 0.59 & 0.00 & 0.21 & 0.39 \\
\hline & 0 \&DF & $1.96^{\mathrm{b}}$ & $0.09^{\mathrm{a}}$ & $0.03^{\mathrm{b}}$ & $0.18^{\mathrm{ab}}$ & $0.02^{\mathrm{e}}$ & $11.87^{\mathrm{a}}$ & $0.09^{\mathrm{abc}}$ & $7.80^{\mathrm{a}}$ & $22.03^{\mathrm{aAB}}$ \\
\hline & $0.8 \mathrm{PE}$ & 0.11 & 0.00 & 0.00 & 0.01 & 0.00 & 0.01 & 0.07 & 0.60 & 0.81 \\
\hline & O 8PW & $2.28^{\mathrm{a}}$ & $0.07^{\mathrm{a}}$ & $0.41^{\mathrm{a}}$ & $0.20^{\mathrm{a}}$ & $0.77^{\mathrm{c}}$ & $8.75^{\mathrm{b}}$ & $0.18^{\mathrm{a}}$ & $9.14^{\mathrm{a}}$ & $21.8^{\mathrm{aA}}$ \\
\hline & $0.8 \mathrm{PW}$ & 0.07 & 0.00 & 0.03 & 0.00 & 0.00 & 0.35 & 0.00 & 0.71 & 1.02 \\
\hline & $C$ & $1.87^{\mathrm{ab}}$ & $0.09^{\mathrm{a}}$ & $0.03^{\mathrm{b}}$ & $0.21^{\mathrm{a}}$ & $0.56^{\mathrm{b}}$ & $11.54^{\mathrm{ab}}$ & $0.05^{\mathrm{d}}$ & $6.24^{\mathrm{d}}$ & $20.58^{\mathrm{bBC}}$ \\
\hline & $c$ & 0.06 & 0.00 & 0.00 & 0.01 & 0.04 & 0.86 & 0.00 & 0.13 & 0.69 \\
\hline & $0 \triangle \mathrm{PF}$ & $1.80^{\mathrm{b}}$ & $0.08^{\mathrm{a}}$ & $0.03^{\mathrm{b}}$ & $0.19^{\mathrm{ab}}$ & $0.03^{\mathrm{d}}$ & $11.46^{\mathrm{ab}}$ & $0.14^{\mathrm{b}}$ & $7.73^{\mathrm{b}}$ & $21.44^{\mathrm{abA}}$ \\
\hline & $0.4 \mathrm{PE}$ & 0.01 & 0.00 & 0.00 & 0.01 & 0.00 & 0.93 & 0.01 & 0.16 & 1.11 \\
\hline 13 & $0.4 \mathrm{PW}$ & $1.87^{\mathrm{ab}}$ & $0.08^{\mathrm{a}}$ & $0.03^{\mathrm{b}}$ & $0.18^{\mathrm{ab}}$ & $1.12^{\mathrm{a}}$ & $12.68^{\mathrm{a}}$ & $0.04^{\mathrm{d}}$ & $6.82^{c}$ & $22.81^{\mathrm{aA}}$ \\
\hline & & 0.01 & 0.00 & 0.00 & 0.01 & 0.03 & 0.26 & 0.00 & 0.2 & 0.42 \\
\hline & & $2.01^{\mathrm{ab}}$ & $0.08^{\mathrm{a}}$ & $0.02^{\mathrm{c}}$ & $0.19^{\mathrm{ab}}$ & $0.56^{\mathrm{b}}$ & $11.92^{\mathrm{ab}}$ & $0.19^{\mathrm{a}}$ & $7.79^{b}$ & $22.75^{\mathrm{aBC}}$ \\
\hline & $0.8 \mathrm{PE}$ & 0.11 & 0.01 & 0.00 & 0.01 & 0.06 & 0.51 & 0.01 & 0.09 & 0.52 \\
\hline & $08 \mathrm{E}$ & $2.12^{\mathrm{a}}$ & $0.09^{\mathrm{a}}$ & $0.17^{\mathrm{a}}$ & $0.17^{\mathrm{b}}$ & $0.39^{c}$ & $10.27^{\mathrm{b}}$ & $0.09^{c}$ & $8.91^{\mathrm{a}}$ & $22.18^{\mathrm{abA}}$ \\
\hline & $0.8 \mathrm{PW}$ & 0.16 & 0.01 & 0.01 & 0.01 & 0.01 & 0.64 & 0.00 & 0.12 & 1.60 \\
\hline & & $2.10^{\mathrm{b}}$ & $0.09^{\mathrm{a}}$ & $0.04^{\mathrm{d}}$ & $0.21^{\mathrm{a}}$ & $0.82^{\mathrm{d}}$ & $10.48^{\mathrm{ab}}$ & $0.05^{\mathrm{c}}$ & $7.61^{\mathrm{a}}$ & $21.38^{\mathrm{abB}}$ \\
\hline & $\mathrm{C}$ & 0.15 & 0.01 & 0.01 & 0.01 & 0.02 & 0.47 & 0.00 & 0.29 & 0.52 \\
\hline & & $2.72^{\mathrm{a}}$ & $0.08^{\mathrm{ab}}$ & $0.42^{\mathrm{a}}$ & $0.21^{\mathrm{a}}$ & $1.02^{\mathrm{c}}$ & $9.61^{\mathrm{b}}$ & $0.11^{\mathrm{ab}}$ & $7.51^{\mathrm{a}}$ & $21.66^{\mathrm{abA}}$ \\
\hline & $0.4 \mathrm{PE}$ & 0.16 & 0.00 & 0.03 & 0.02 & 0.01 & 0.25 & 0.00 & 0.46 & 0.59 \\
\hline & & $1.90^{\mathrm{b}}$ & $0.09^{\mathrm{a}}$ & $0.03^{\mathrm{d}}$ & $0.22^{\mathrm{a}}$ & $1.19^{\mathrm{a}}$ & $11.36^{\mathrm{a}}$ & $0.10^{\mathrm{b}}$ & $6.75^{\mathrm{a}}$ & $21.62^{\mathrm{abBC}}$ \\
\hline 15 & $0.4 \mathrm{PW}$ & 0.07 & 0.01 & 0.00 & 0.02 & 0.02 & 0.83 & 0.00 & 0.28 & 0.62 \\
\hline & 0 8PF & $2.91^{\mathrm{a}}$ & $0.07^{\mathrm{b}}$ & $0.20^{\mathrm{c}}$ & $0.22^{\mathrm{a}}$ & $1.08^{\mathrm{bc}}$ & $9.73^{\mathrm{b}}$ & $0.12^{\mathrm{a}}$ & $6.86^{\mathrm{a}}$ & $21.18^{\mathrm{bABC}}$ \\
\hline & $0.8 \mathrm{PE}$ & 0.16 & 0.00 & 0.01 & 0.02 & 0.06 & 0.04 & 0.00 & 0.52 & 0.35 \\
\hline & & $2.68^{\mathrm{a}}$ & $0.08^{\mathrm{ab}}$ & $0.36^{\mathrm{b}}$ & $0.19^{\mathrm{a}}$ & $1.10^{\mathrm{b}}$ & $10.28^{\mathrm{ab}}$ & $0.11^{\mathrm{ab}}$ & $7.75^{\mathrm{a}}$ & $22.54^{\mathrm{aA}}$ \\
\hline & $0.8 \mathrm{PW}$ & 0.24 & 0.00 & 0.01 & 0.01 & 0.03 & 0.35 & 0.01 & 0.40 & 0.13 \\
\hline
\end{tabular}


Table 4. Changes in the ratio and percentage of selected fatty acids in sardine treated with/without water or ethanolic extract of propolis during chill storage

\begin{tabular}{|c|c|c|c|c|c|c|c|c|}
\hline Days & & PUFA/SFA & n3 & n6 & $\mathrm{n} 6 / \mathrm{n} 3$ & DHA & EPA & DHA/EPA \\
\hline \multirow{2}{*}{0} & \multirow{2}{*}{$\mathrm{C}$} & $0.51^{\mathrm{xA}}$ & $18.34^{\mathrm{ABC}}$ & $3.07^{\mathrm{A}}$ & $0.17^{\mathrm{A}}$ & $7.16^{\mathrm{AB}}$ & $10.95^{\mathrm{AB}}$ & $0.65^{\mathrm{AB}}$ \\
\hline & & $0.02^{y}$ & 1.06 & 0.31 & 0.03 & 0.66 & 0.40 & 0.04 \\
\hline \multirow{10}{*}{4} & \multirow{2}{*}{$\mathrm{C}$} & $0.46^{\mathrm{bA}}$ & $16.59^{\mathrm{C}}$ & $2.88^{\mathrm{bA}}$ & $0.17^{\mathrm{aA}}$ & $6.22^{\mathrm{bB}}$ & $10.11^{\mathrm{bB}}$ & $0.62^{\mathrm{bAB}}$ \\
\hline & & 0.05 & 1.21 & 0.15 & 0.02 & 0.33 & 0.91 & 0.02 \\
\hline & \multirow{2}{*}{$0.4 \mathrm{PE}$} & $0.57^{\mathrm{aA}}$ & $19.80^{\mathrm{aA}}$ & $2.49^{\mathrm{cC}}$ & $0.13^{\mathrm{bC}}$ & $8.19^{\mathrm{aA}}$ & $11.58^{\mathrm{ab} A B}$ & $0.71^{\mathrm{ab} A \mathrm{~B}}$ \\
\hline & & 0.01 & 0.70 & 0.04 & 0.01 & 0.22 & 0.91 & 0.07 \\
\hline & \multirow{2}{*}{$0.4 \mathrm{PW}$} & $0.58^{\mathrm{aA}}$ & $19.75^{\mathrm{aA}}$ & $2.92^{\mathrm{bB}}$ & $0.15^{\mathrm{abC}}$ & $7.49^{\mathrm{abB}}$ & $12.22^{\mathrm{aAB}}$ & $0.61^{\mathrm{bB}}$ \\
\hline & & 0.00 & 0.11 & 0.01 & 0.00 & 0.11 & 0.00 & 0.01 \\
\hline & \multirow{2}{*}{$0.8 \mathrm{PE}$} & $0.57^{\mathrm{aA}}$ & $19.23^{\mathrm{aAB}}$ & $3.22^{\mathrm{aB}}$ & $0.17^{\mathrm{aBC}}$ & $7.34^{\mathrm{bAB}}$ & $11.84^{\mathrm{ab}}$ & $0.62^{\mathrm{bC}}$ \\
\hline & & 0.04 & 1.10 & 0.07 & 0.01 & 0.11 & 0.99 & 0.04 \\
\hline & \multirow{2}{*}{$0.8 \mathrm{PW}$} & $0.51^{\mathrm{abB}}$ & $18.42^{\mathrm{abA}}$ & $2.47^{\mathrm{cC}}$ & $0.13^{\mathrm{bC}}$ & $8.03^{\mathrm{abABC}}$ & $10.35^{\mathrm{abB}}$ & $0.78^{\mathrm{aB}}$ \\
\hline & & 0.03 & 0.81 & 0.08 & 0.01 & 0.54 & 0.27 & 0.03 \\
\hline \multirow{10}{*}{7} & \multirow{2}{*}{ C } & $0.48^{\mathrm{aA}}$ & $18.62^{\mathrm{aAB}}$ & $1.12^{\mathrm{dC}}$ & $0.06^{\mathrm{dB}}$ & $7.59^{\mathrm{abcA}}$ & $11.00^{\mathrm{ab} A B}$ & $0.69^{\mathrm{abcA}}$ \\
\hline & & 0.02 & 0.32 & 0.01 & 0.00 & 0.16 & 0.48 & 0.04 \\
\hline & \multirow{2}{*}{$0.4 \mathrm{PE}$} & $0.52^{\mathrm{aA}}$ & $18.69^{\mathrm{aA}}$ & $1.82^{\mathrm{bE}}$ & $0.10^{\mathrm{bD}}$ & $7.23^{\mathrm{bcB}}$ & $11.44^{\mathrm{abAB}}$ & $0.63^{\mathrm{bcB}}$ \\
\hline & & 0.03 & 1.10 & 0.10 & 0.00 & 0.70 & 0.40 & 0.04 \\
\hline & \multirow{2}{*}{$0.4 \mathrm{PW}$} & $0.52^{\mathrm{aB}}$ & $18.94^{\mathrm{aAB}}$ & $1.76^{\mathrm{bC}}$ & $0.09^{\mathrm{bcD}}$ & $6.77^{\mathrm{cC}}$ & $12.15^{\mathrm{aAB}}$ & $0.56^{\mathrm{cB}}$ \\
\hline & & 0.02 & 0.23 & 0.07 & 0.00 & 0.01 & 0.22 & 0.01 \\
\hline & \multirow{2}{*}{$0.8 \mathrm{PE}$} & $0.53^{\mathrm{aAB}}$ & $19.38^{\mathrm{aAB}}$ & $2.53^{\mathrm{aC}}$ & $0.13^{\mathrm{aDE}}$ & $8.20^{\mathrm{abA}}$ & $11.15^{\mathrm{abAB}}$ & $0.74^{\mathrm{abABC}}$ \\
\hline & & 0.03 & 0.61 & 0.08 & 0.01 & 0.23 & 0.84 & 0.08 \\
\hline & QPWW & $0.51^{\mathrm{aB}}$ & $19.29^{\mathrm{aA}}$ & $1.50^{\mathrm{cD}}$ & $0.08^{\mathrm{c}}$ & $8.47^{\mathrm{aABC}}$ & $10.79^{\mathrm{bB}}$ & $0.79^{\mathrm{aB}}$ \\
\hline & $0.8 \mathrm{PW}$ & 0.01 & 0.18 & 0.06 & 0.00 & 0.50 & 0.33 & 0.07 \\
\hline & $C$ & $0.51^{\mathrm{bA}}$ & $17.92^{\mathrm{aABC}}$ & $3.04^{\mathrm{bA}}$ & $0.17^{\mathrm{abA}}$ & $7.03^{\mathrm{cAB}}$ & $10.87^{\mathrm{ab} A B}$ & $0.65^{\mathrm{bAB}}$ \\
\hline & $c$ & 0.01 & 0.36 & 0.19 & 0.01 & 0.30 & 0.06 & 0.02 \\
\hline & $04 \mathrm{PF}$ & $0.57^{\mathrm{abA}}$ & $19.38^{\mathrm{aA}}$ & $3.22^{\mathrm{bB}}$ & $0.17^{\mathrm{abB}}$ & $7.30^{\mathrm{cAB}}$ & $11.70^{\mathrm{aAB}}$ & $0.62^{\mathrm{bB}}$ \\
\hline & $0.41 \mathrm{~L}$ & 0.02 & 0.76 & 0.05 & 0.00 & 0.22 & 0.52 & 0.01 \\
\hline 9 & $04 \mathrm{PW}$ & $0.56^{\mathrm{abAB}}$ & $18.99^{\mathrm{aAB}}$ & $3.21^{\mathrm{bA}}$ & $0.17^{\mathrm{abAB}}$ & $8.31^{\mathrm{aA}}$ & $10.67^{\mathrm{bC}}$ & $0.78^{\mathrm{aA}}$ \\
\hline 9 & $0.4 \mathrm{PW}$ & 0.01 & 0.44 & 0.16 & 0.01 & 0.05 & 0.49 & 0.04 \\
\hline & $08 \mathrm{PF}$ & $0.62^{\mathrm{abA}}$ & $19.62^{\mathrm{aAB}}$ & $3.83^{\mathrm{aA}}$ & $0.19^{\mathrm{abB}}$ & $8.07^{\mathrm{abA}}$ & $11.53^{\mathrm{bAB}}$ & $0.69^{\mathrm{bAB}}$ \\
\hline & $0.8 \mathrm{PE}$ & 0.04 & 0.16 & 0.25 & 0.02 & 0.40 & 0.18 & 0.02 \\
\hline & 0 8PW & $0.55^{\mathrm{abA}}$ & $19.08^{\mathrm{aB}}$ & $3.07^{\mathrm{bB}}$ & $0.16^{\mathrm{bB}}$ & $7.34^{\mathrm{bcC}}$ & $11.60^{\mathrm{aA}}$ & $0.63^{\mathrm{bC}}$ \\
\hline & $0.8 \mathrm{PW}$ & 0.00 & 0.61 & 0.01 & 0.00 & 0.35 & 0.27 & 0.02 \\
\hline & & $0.54^{\mathrm{aA}}$ & $19.71^{\mathrm{aA}}$ & $3.01^{\mathrm{aA}}$ & $0.15^{\mathrm{aA}}$ & $7.81^{\mathrm{aA}}$ & $11.87^{\mathrm{aA}}$ & $0.66^{\mathrm{bA}}$ \\
\hline & C & 0.00 & 0.25 & 0.00 & 0.00 & 0.68 & 0.43 & 0.08 \\
\hline & & $0.53^{\mathrm{aA}}$ & $19.23^{\mathrm{aA}}$ & $2.18^{\mathrm{bD}}$ & $0.11^{\mathrm{bCB}}$ & $7.87^{\mathrm{aAB}}$ & $11.32^{\mathrm{aAB}}$ & $0.70^{\mathrm{bAB}}$ \\
\hline & $0.4 \mathrm{PE}$ & 0.01 & 0.33 & 0.09 & 0.01 & 0.28 & 0.08 & 0.02 \\
\hline 11 & 04 DW & $0.54^{\mathrm{aAB}}$ & $19.23^{\mathrm{aA}}$ & $2.96^{\mathrm{aB}}$ & $0.15^{\mathrm{aBC}}$ & $8.30^{\mathrm{aA}}$ & $10.90^{\mathrm{aC}}$ & $0.76^{\mathrm{bA}}$ \\
\hline 11 & $0.4 \mathrm{PW}$ & 0.02 & 0.40 & 0.01 & 0.00 & 0.21 & 0.59 & 0.06 \\
\hline & O 8PF & $0.55^{\mathrm{aAB}}$ & $19.69^{\mathrm{aA}}$ & $2.07^{\mathrm{bD}}$ & $0.11^{\mathrm{bE}}$ & $7.80^{\mathrm{aAB}}$ & $11.87^{\mathrm{aA}}$ & $0.66^{\mathrm{bBC}}$ \\
\hline & $0.8 \mathrm{PE}$ & 0.02 & 0.61 & 0.11 & 0.00 & 0.60 & 0.01 & 0.05 \\
\hline & $08 P W$ & $0.51^{\mathrm{aB}}$ & $18.30^{\mathrm{aB}}$ & $3.12^{\mathrm{aB}}$ & $0.17^{\mathrm{aB}}$ & $9.14^{\mathrm{aA}}$ & $8.75^{\mathrm{bC}}$ & $1.04^{\mathrm{aA}}$ \\
\hline & $0.8 \mathrm{PW}$ & 0.01 & 1.09 & 0.07 & 0.01 & 0.71 & 0.35 & 0.04 \\
\hline & & $0.50^{\mathrm{bcA}}$ & $17.81^{\mathrm{cBC}}$ & $2.51^{\mathrm{cB}}$ & $0.14^{\mathrm{abA}}$ & $6.24^{\mathrm{dB}}$ & $11.54^{\mathrm{abA}}$ & $0.54^{\mathrm{cB}}$ \\
\hline & $\mathrm{C}$ & 0.01 & 0.74 & 0.03 & 0.01 & 0.13 & 0.86 & 0.05 \\
\hline & $04 \mathrm{PE}$ & $0.45^{\mathrm{cB}}$ & $19.22^{\mathrm{bA}}$ & $1.91^{\mathrm{dE}}$ & $0.10^{\mathrm{cD}}$ & $7.73^{\mathrm{bAB}}$ & $11.46^{\mathrm{abAB}}$ & $0.68^{\mathrm{bB}}$ \\
\hline & $0.4 \mathrm{PE}$ & 0.04 & 1.08 & 0.02 & 0.00 & 0.16 & 0.93 & 0.04 \\
\hline 13 & $04 \mathrm{PW}$ & $0.58^{\mathrm{aA}}$ & $19.52^{\mathrm{abA}}$ & $3.07^{\mathrm{aAB}}$ & $0.16^{\mathrm{aBC}}$ & $6.82^{\mathrm{cC}}$ & $12.68^{\mathrm{aA}}$ & $0.54^{\mathrm{cB}}$ \\
\hline 10 & 0.412 & 0.01 & 0.46 & 0.02 & 0.00 & 0.20 & 0.26 & 0.00 \\
\hline & $08 \mathrm{E}$ & $0.46^{\mathrm{cB}}$ & $19.73^{\mathrm{aC}}$ & $2.65^{\mathrm{bC}}$ & $0.13^{\mathrm{bcCD}}$ & $7.79^{\mathrm{bAB}}$ & $11.92^{\mathrm{bB}}$ & $0.65^{\mathrm{bA}}$ \\
\hline & 0.8 & 0.01 & 0.60 & 0.05 & 0.01 & 0.09 & 0.51 & 0.03 \\
\hline & 0 QPW & $0.54^{\mathrm{abAB}}$ & $19.61^{\mathrm{aA}}$ & $2.65^{\mathrm{bC}}$ & $0.14^{\mathrm{abC}}$ & $8.95 \mathrm{a}^{\mathrm{AB}}$ & $10.50^{\mathrm{bB}}$ & $0.85^{\mathrm{aB}}$ \\
\hline & $0.8 \mathrm{PW}$ & 0.01 & 0.37 & 0.09 & 0.00 & 0.06 & 0.32 & 0.02 \\
\hline & & $0.52^{\mathrm{aA}}$ & $18.39^{\mathrm{aABC}}$ & $2.93^{\mathrm{bA}}$ & $0.16^{\mathrm{cA}}$ & $7.71^{\mathrm{aA}}$ & $10.65^{\mathrm{abAB}}$ & $0.72^{\mathrm{aA}}$ \\
\hline & C & 0.01 & 0.37 & 0.09 & 0.01 & 0.14 & 0.23 & 0.00 \\
\hline & & $0.52^{\mathrm{aA}}$ & $17.79^{\mathrm{bA}}$ & $3.76^{\mathrm{aA}}$ & $0.21^{\mathrm{abA}}$ & $7.67^{\mathrm{aAB}}$ & $9.69^{\mathrm{bB}}$ & $0.79^{\mathrm{aA}}$ \\
\hline & $0.4 \mathrm{PE}$ & 0.00 & 0.37 & 0.08 & 0.01 & 0.23 & 0.12 & 0.01 \\
\hline & & $0.53^{\mathrm{aB}}$ & $18.13^{\mathrm{aB}}$ & $3.17^{\mathrm{bA}}$ & $0.17^{\mathrm{bcA}}$ & $6.75^{\mathrm{bC}}$ & $11.36^{\mathrm{aBC}}$ & $0.60^{\mathrm{bB}}$ \\
\hline 15 & $0.4 \mathrm{PW}$ & 0.03 & 0.56 & 0.08 & 0.00 & 0.28 & 0.83 & 0.07 \\
\hline & 0 8PE & $0.51^{\mathrm{aAB}}$ & $16.79^{\mathrm{bC}}$ & $4.06^{\mathrm{aA}}$ & $0.24^{\mathrm{aA}}$ & $6.86^{\mathrm{abB}}$ & $9.73^{\mathrm{bB}}$ & $0.70^{\mathrm{abABC}}$ \\
\hline & $0.8 \mathrm{PE}$ & 0.00 & 0.54 & 0.22 & 0.02 & 0.52 & 0.04 & 0.05 \\
\hline & & 0.51 & $18.38^{\mathrm{aA}}$ & $3.86^{\mathrm{aA}}$ & $0.21^{\mathrm{abA}}$ & $7.75^{\mathrm{aBC}}$ & $10.28^{\mathrm{abB}}$ & $0.76^{\mathrm{aB}}$ \\
\hline & $0.8 \mathrm{PW}$ & 0.00 & 0.06 & 0.21 & 0.01 & 0.40 & 0.35 & 0.06 \\
\hline
\end{tabular}

${ }^{\mathrm{x}}$ Average value, ${ }^{\mathrm{y}}$ Standard deviation $(\mathrm{n}=3)$. Means followed by different letters are significantly different $(\mathrm{P}<0.05)$ among groups $\left({ }^{\mathrm{a}-\mathrm{d}}\right)$ and during storage $^{\text {(A-D) }}$. C: Control group, 0.4PE: group treated with ethanolic extract of propolis at doses of $0.4 \%, 0.8 \mathrm{PE}$ : group treated with ethanolic extract of propolis at doses of $0.8 \%, 0.4 \mathrm{PW}$ : group treated with water extract of propolis at doses of $0.4 \%, 0.4 \mathrm{PW}$ : group treated with water extract of propolis at doses of $0.8 \%$. 
Eicosapentaenoic acid (EPA; C20:5 n3) and docosahexaenoic acid (DHA; C22:6 n3) were the most important PUFA found at high levels in sardine fillets, followed by linoleic (C18:2 n6) and arachidonic acid (C20:4 n6). Similarly, the fatty acid composition of sardine represented a high content of PUFAs dominated by n-3 fatty acids EPA and DHA with maximum rates of 15.75 and $33.42 \%$, respectively (Benguendouz et al., 2017). EPA along with DHA are the key omega-3 fatty acids which excluded in high levels in fish oil. These fatty acids originate in unicellular phytoplanktons and seaweeds and accumulate in fish (Sahena et al., 2009). The evidence base supports a dietary recommendation of $500 \mathrm{mg} /$ day of EPA and DHA for cardiovascular disease risk reduction (Gebauer et al., 2006). EPA and DHA represented 10.95 and $7.16 \%$ of PUFA at 0 day. EPA and DHA were the most affected PUFA during refrigerated storage (Tenyang et al., 2017). Marked decreases in unsaturated fatty acids, especially EPA and DHA were observed as the storage time increased. Those changes indicated that lipid oxidation occurred in sardine muscle during iced storage (Chaijan et al., 2006). Ucak (2018) found that the oxidation level is lower and the most effective at doses of $1000 \mathrm{mg} / \mathrm{kg}$ propolis ethanolic extract added in fish oil. In the current study, there was no significant differences in EPA and DHA between control and treated groups during storage apart from at $4^{\text {th }}$ and $11^{\text {th }}$ day $(\mathrm{P}>0.05)$. Water propolis extracts at doses of 0.4 and $0.8 \%$ had higher content EPA than control at $4^{\text {th }}$ and $11^{\text {th }}$ day, respectively. At 11 day, $0.8 \mathrm{PW}$ group had lower EPA content than other treated group. DHA content of control group was the lowest at 4 and $13^{\text {th }}$ day that of treated groups with propolis extracts. DHA did not differ statistically between control and treated group at 7, 11 and 15 days. $0.4 \mathrm{PW}$ group had lower content of DHA than $0.8 \mathrm{PW}$ group at $7^{\text {th }}$ day, but it was higher at $9^{\text {th }}$ day. Chardonnay and black raspberry seed extracts in preserving EPA and DHA may differ to that in suppressing the overall lipid oxidation in the fish oil, and this observation may be partially explained by the different polarity of the antioxidants in each antioxidant preparations, which may lead to the different distribution of anti-oxidative components in the oil (Luther et al., 2007).

Total PUFA content sardine was $21.59 \%$ at the beginning. Although slight decreases were observed on total PUFA content of control group, this decreases was not statistically significant $(\mathrm{P}>0.05)$ apart from $4^{\text {th }}$ day of storage. PUFA levels in vacuum-packed tuna flesh stored at $0^{\circ} \mathrm{C}$ remained unchanged during the three first days (from $41.32 \%$ to $41.28 \%$ ), thereafter, a significant decrease $(\mathrm{P}<0.05)$ was showed in $6^{\text {th }}$ day $(39.89 \%)$ and in $12^{\text {th }}$ day $(38.74 \%)$. In dried thyme treated samples, PUFAs levels remain significantly constant from day $0(41.32 \%)$ to day $15(41.05 \%)$, thereafter, a significant decrease was showed at the end of storage (day 18) to reach $39.38 \%$ (Selmi and Sadok, 2008). In the current study, total PUFA also did not change considerably for $0.4 \mathrm{PE}$ group, whilst significant changes were observed for $0.4 \mathrm{PW}$ and $0.8 \mathrm{PE}$ group at 7 and $15^{\text {th }}$ days, and $0.8 \mathrm{PW}$ group at $11^{\text {th }}$ days. At 4 and 9 days of storage, control group had the lowest content of PUFA than treated group. Moreover, 0.8PE group had also higher amount of PUFA than that of control group at 7 and 13 days. PUFA content of $0.8 \mathrm{PE}$ and $0.8 \mathrm{PW}$ group was higher than that of other groups at 9 days of storage. The higher PUFA content of treated groups implicated antioxidant properties of propolis extracts. Similarly, the treatment of $\alpha$-tocopherol-rosemary mixture $(0.05 \%+0.02 \%)$ of sardine oil inhibited oxidation of PUFA (Wada and Fang, 1992).

PUFA/SFA ratio of control group did not alter significantly during storage, represented ranging value of 0.46-0.60 (Table 4). This value was lower than reported values (0.8-1.1) for sardine (Özogul and Özogul, 2007; Benguendouz et al., 2017). Significant differences in PUFA/SFA ratio were observed for treated group during storage $(\mathrm{P}<0.05)$. However, there was not significant differences among groups at 7, 9, 11 and $15^{\text {th }}$ days. Treated groups apart from $0.8 \mathrm{PW}$ had higher PUFA/SFA ratio at $4^{\text {th }}$ day than control.

Total n3 amount of sardine was $18.34 \%$ at the beginning of storage. Zlatanos and Laskaridis (2007) reported higher total $\mathrm{n} 3$ content in sardine caught from various seasons (30.49 and 40.86\%). Significant differences were observed among groups at 4,13 and $15^{\text {th }}$ day. At 4 and $13^{\text {th }}$ day, propolis treated group had considerably higher $\mathrm{n} 3$ content than control $(\mathrm{P}<0.05)$. Ethanolic propolis extracts included lower $n 3$ content than other groups at $15^{\text {th }}$ day. Total $n 6$ content of sardine was lower than total $\mathrm{n} 3$ amount which is in agreement with those reported by other studies (Passi et al., 2002; GarciaArias et al., 2003; De Leonardis and Macciola, 2004; Zlatanos and Laskaridis, 2007). These results point out the high quality of sardine fillet-fat from a cardiovascular point of view (Garcia-Arias et al., 2003)

The scientific evidence is strong for decreasing the $n-6$ and increasing the n-3 intake to improve health throughout the life cycle (Simopoulos, 2001). The ratio of $n-6 / n-3$ around $1: 1$ to 2:1 is considered good for human health; however, any increase in the ratio is usually attributed to human diet (Bulla et al., 2011). Initial n6/n3 ratio in sardine fillet was 0.17 . At 4 , 11 and $13^{\text {th }}$ days, $0.4 \mathrm{PE}$ group comprised lower $\mathrm{n} 6 / \mathrm{n} 3$ ratio. However, control group possessed lower $n 6 / n 3$ ratio than treated group at 7 and $15^{\text {th }}$ days.

General recommendation for daily intakes of DHA/EPA is $0.5 \mathrm{~g} /$ day for infants and $1 \mathrm{~g} /$ day for adults and patients with heart disease (Kris-Etherton et al., 2002). The ratio of DHA/EPA in sardine was found in the range of 0.56 and $1.04 \%$ during storage. $0.8 \mathrm{PW}$ groups generally contained the highest ratio of DHA/EPA compared to other groups.

\section{Conclusion}

Sardine meat was rich in palmitic acid, palmitoleic acid, myristic acid (C14:0), and PUFA of EPA and DHA. In studies conducted to date, propolis have been reported to have strong antioxidant properties. However, there are not enough studies regarding their effect on fish fatty acids during storage. The study results showed that the effect of water and ethanolic propolis extracts on fatty acids varied with the concentration of propolis and storage days. However, ethanolic and water extracts especially at doses of $0.8 \%$ seemed to effective to maintain stability of PUFA content in sardine meat during chill storage. 


\section{Acknowledgements}

The author would like to thank to Yesim Özogul and colleagues who provided support in carrying out the work.

\section{References}

Ahn MR, Kumazawa S, Usui Y, Nakamura J, Matsuka M, Zhu F, Nakayama T. 2007. Antioxidant activity and constituents of propolis collected in various areas of China. Food Chem., 101: 1383-1392. DOI:10.1016/j.foodchem.2006.03.045.

Ali M, Imran M, Nadeem M, Khan MK., Sohaib M, Suleria HAR, Bashir R. 2019. Oxidative stability and sensoric acceptability of functional fish meat product supplemented with plant-based polyphenolic optimal extracts. Lipids Health Dis., 18(35): 1-16. DOI:10.1186/s12944-019-0982-y.

Benguendouz A, Bouderoua K, Bouterfa A, Belabes M, Bekada A. Sioriki E, Zabetakis I. 2017. Fatty acid profile and assessment of heavy metals content of Sardina pilchardus captured in the Algerian coast. Iran J Fish Sci., 16: 10211029.

Bligh EG, Dyer WJ. 1959. A rapid method of total lipid extraction and purification. Can J Biochem Physiol,. 37: 911- 917.

Boufadi Y, Soubhye J, Riazi A, Rousseau A, Vanhaeverbeek M, Nève J, Van Antwerpen P. 2014. Characterization and antioxidant properties of six Algerian propolis extracts: ethyl acetate extracts inhibit myeloperoxidase activity. Int $\mathrm{J}$ Mol Sci., 15: 2327-2345. DOI:10.3390/ijms 15022327.

Bulla MK, Simionato JI, Matsushita M, Coro FA, Shimokomaki M, Visentainer JV, Souza NE. 2011. Proximate composition and fatty acid profile of raw and roasted salt-dried sardines (Sardinella brasiliensis). Food Nutr Sci,, 2: 440-3. DOI:10.4236/fns.2011.25062.

Chaijan M, Benjakul S, Visessanguan W, Faustman C. 2006. Changes of lipids in sardine (Sardinella gibbosa) muscle during iced storage. Food Chem., 99: 83-91. DOI:10.1016/j.foodchem.2005.07.022.

De Leonardis A, Macciola,V. 2004. A study on the lipid fraction of Adriatic sardine filets (Sardina pilchardus). Nahrung., 48: 209-212. DOI:10.1002/food.200300408.

Garcia-Arias MT, Pontes EÁ, Garcia-Linares MC, GarciaFernandez MC, Sanchez-Muniz FJ. 2003. Cooking-freezingreheating (CFR) of sardine (Sardina pilchardus) fillets. Effect of different cooking and reheating procedures on the proximate and fatty acid compositions. Food Chem., 83: 349356. DOI:10.1016/S0308-8146(03)00095-5.

Gebauer SK, Psota TL, Harris WS, Kris-Etherton PM. 2006. n-3 fatty acid dietary recommendations and food sources to achieve essentiality and cardiovascular benefits. Am J Clin Nutr., 83: 1526S-1535S. DOI:10.1093/ajcn/83.6.1526S.

Gutiérrez-Cortés C, Suarez-Mahecha H. 2014. Antimicrobial activity of propolis and its effect on the physicochemical and sensoral characteristics in sausages. Vitae, 21: 90-96.

Hernandez Zarate MS, Abraham Juarez MDR, Ceron Garcia A, Ozuna Lopez C, Gutierrez Chavez AJ, Segoviano Garfias JDN, Avila Ramos F. 2018. Flavonoids, phenolic content, and antioxidant activity of propolis from various areas of Guanajuato, Mexico. Food Sci., Tech., 38: 210-215.

Huang S, Zhang CP, Wang K, Li G, Hu FL. 2014. Recent advances in the chemical composition of propolis. Molecules, 19: 19610-19632. DOI:10.3390/molecules191219610.

Ichihara KI, Shibahara A, Yamamoto K, Nakayama T. 1996. An improved method for rapid analysis of the fatty acids of glycerolipids. $\quad$ Lipids, 31 535-539. DOI:10.1007/BF02522648

Kris-Etherton PM, Harris WS, Appel LJ. 2002. Fish consumption, fish oil, omega-3 fatty acids, and cardiovascular disease. Circulation, 106: 2747-2757. DOI:10.1161/01.cir.0000038493.65177.94.
Kročko M, Bobko M, Bučko O, Čanigová M, Ducková V. 2014. Sensory quality, colour and oxidative stability of cured cooked ham with propolis extract. Potravinarstvo, 8: 102106. DOI: $10.5219 / 365$.

Luther M, Parry J, Moore J, Meng J, Zhang Y, Cheng Z, Yu L. 2007. Inhibitory effect of Chardonnay and black raspberry seed extracts on lipid oxidation in fish oil and their radical scavenging and antimicrobial properties. Food Chem., 104:1065-1073. DOI:10.1016/j.foodchem.2007.01.034.

Metillo EB, Aspiras-Eya AA. 2014. Fatty acids in six small pelagic fish species and their crustacean prey from the Mindanao Sea, Southern Philippines. Trop Life Sci Res., 25: 105-115.

Özogul Y, Özogul F. 2007. Fatty acid profiles of commercially important fish species from the Mediterranean, Aegean and Black Seas. Food Chem., 100: 1634-1638. DOI:10.1016/j.foodchem.2005.11.047..

Passi S, Cataudella S, Di Marco P, De Simone F, Rastrelli L. 2002. Fatty acid composition and antioxidant levels in muscle tissue of different Mediterranean marine species of fish and shellfish. J Agric Food Chem., 50: 7314-7322. DOI:10.1021/jf020451y.

Sahena F, Zaidul I, Jinap S, Saari N, Jahurul H, Abbas K, Norulaini N. 2009. PUFAs in fish: Extraction, fractionation, importance in health. Compr Rev Food Sci., 8:59-74. DOI:10.1111/j.1541-4337.2009.00069.x.

Selmi S, Sadok S. 2008. The effect of natural antioxidant (Thymus vulgaris Linnaeus) on flesh quality of tuna (Thunnus thynnus (Linnaeus)) during chilled storage. Pan Am J Aquat Sci., 3: 36-45.

Simopoulos AP. 2001. N-3 fatty acids and human health: Defining strategies for public policy. Lipids, 36: 83-89.

Tenyang N, Womeni HM, Tiencheu B, Villeneuve P, Linder M. 2017. Effect of refrigeration time on the lipid oxidation and fatty acid profiles of catfish (Arius maculatus) commercialized in Cameroon. Grasas Aceites, 68: 1-9. DOI:10.3989/gya.0335161.

Touzani S, Al-Waili N, El Menyiy N, Filipic B, Pereyra A, Arabi IE, Lyoussi B. 2018. Chemical analysis and antioxidant content of various propolis samples collected from different regions and their impact on antimicrobial activities. Asian Pac J Trop Med., 11: 436-442. DOI:10.4103/19957645.237188.

Ucak I. 2018. Determination of the lipid oxidation level in fish oil enriched with propolis extract. J Food., 43: 523-532.

van der Meeren, Olsen RE, Hamre K, Fyhn HJ. 2008. Biochemical composition of copepods for evaluation of feed quality in production of juvenile marine fish. Aquaculture, 274: 375-397. DOI:10.1016/j.aquaculture.2007.11.041.

Vargas-Sánchez RD, Torrescano-Urrutia GR, Acedo-Félix E, Carvajal-Millán E, González-Córdova AF, Vallejo-Galland B, Torrez-Lianez MJ, Sanchez-Escalante A. 2014. Antioxidant and antimicrobial activity of commercial propolis extract in beef patties. J Food Sci., 79: 1499-1504. DOI:10.1111/1750-3841.12533

Wada S, Fang X. 1992. The synergistic antioxidant effect of rosemary extract and $\alpha$-tocopherol in sardine oil model system and frozen-crushed fish meat. J Food Process Pres., 16: 263-274. DOI:10.1111/j.1745-4549.1992.tb00207.x

Yamamoto Y, Imose K. 1989. Changes in fatty acid composition in sardines (Sardinops melanosticta) with cooking and refrigerated storage. J Nutr Sci Vitaminol., 35: 39-47.

Zheng YZ, Deng G, Liang Q, Chen DF, Guo R, Lai RC. 2017. Antioxidant activity of quercetin and its glucosides from propolis. Sci Rep, 7: 7543. doi:10.1038/s41598-017-08024-8

Zlatanos S, Laskaridis K. 2007. Seasonal variation in the fatty acid composition of three Mediterranean fish-sardine (Sardina pilchardus), anchovy (Engraulis encrasicholus) and picarel (Spicara smaris). Food Chem., 103: 725-728. DOI:10.1016/j.foodchem.2006.09.013. 